J. DIFFERENTIAL GEOMETRY

85 (2010) 229-269

\title{
CAT(0) AND CAT(-1) FILLINGS OF HYPERBOLIC MANIFOLDS
}

\author{
KoJi FujiWARA \& Jason Fox MANning
}

\begin{abstract}
We give new examples of hyperbolic and relatively hyperbolic groups of cohomological dimension $d$ for all $d \geq 4$ (see Theorem 2.13). These examples result from applying $\mathrm{CAT}(0) / \mathrm{CAT}(-1)$ filling constructions (based on singular doubly warped products) to finite volume hyperbolic manifolds with toral cusps.

The groups obtained have a number of interesting properties, which are established by analyzing their boundaries at infinity by a kind of Morse-theoretic technique, related to but distinct from ordinary and combinatorial Morse theory (see Section 5).
\end{abstract}

\section{Contents}

1. Introduction

1.1. Outline 231

1.2. Acknowledgments 232

2. Statements of results 232

2.1. Cones and fillings 232

2.2. Main results 234

3. Preliminaries 236

3.1. Warped products 237

3.2. The space of directions and the tangent cone 239

3.3. Locally injective logarithms 240

4. The metric construction and proof of Theorem $2.7 \quad 241$

4.1. A model for the singular part 242

4.2. Curvatures in Riemannian warped products 246

4.3. Gluing functions 246

4.4. Nonpositively curved metrics on the partial cones 250

4.5. Proof of Theorem 2.7 and Proposition $2.8 \quad 252$

The first author was partially supported by Grant-in-Aid for Scientific Research (No. 19340013). The second author was partially supported by NSF grants DMS0301954 and DMS-0804369.

Received 01/12/2009. 
4.6. Isolated flats 252

5. Visual boundaries of fillings 254

5.1. The boundary as an inverse limit 254

5.2. Simple connectivity at infinity 264

6. Further questions 265

References 266

\section{Introduction}

In this paper we study some generalizations of the Gromov-Thurston $2 \pi$ theorem $[\mathbf{2 0}, \mathbf{1 2}]$. Informally, the $2 \pi$ theorem states that "most" Dehn fillings of a hyperbolic 3-manifold with cusps admit negatively curved metrics. Moreover, these negatively curved metrics are close approximations of the metric on the original cusped manifold. Grouptheoretically, the fundamental groups of these fillings are relatively hyperbolic (defined below). If all cusps of the original manifold were filled, the fundamental group of the filling is hyperbolic; if moreover the original manifold was finite volume, the filling is an aspherical closed 3manifold.

In the world of coarse geometry, the correct generalizations of fundamental groups of hyperbolic manifolds with and without cusps are relatively hyperbolic and hyperbolic groups, respectively. These generalizations were introduced by Gromov in [22]. We give a quick review of the definitions: A metric space is $\delta$-hyperbolic for $\delta>0$ if all its geodesic triangles are $\delta$-slim, meaning each side of the triangle is contained in the $\delta$-neighborhood of the other two. A space is Gromov hyperbolic if it is $\delta$-hyperbolic for some $\delta$. Groups which are hyperbolic or relatively hyperbolic are those which have particular kinds of actions on Gromov hyperbolic spaces. If $G$ acts by isometries on a Gromov hyperbolic space $X$ properly and cocompactly, then $G$ is said to be hyperbolic. A geometrically finite action of a group $G$ on a Gromov hyperbolic space $X$ is one which satisfies the following conditions:

1) $G$ acts properly and by isometries on $X$.

2) There is a family $\mathcal{H}$ of disjoint horoballs (sub-level sets of so-called horofunctions), preserved by the action.

3) The action on $X \backslash \bigcup \mathcal{H}$ is cocompact.

4) The quotient $X / G$ is quasi-isometric to a wedge of rays.

(See [22] for more details.) If $G$ acts on $X$ geometrically finitely, then $G$ is said to be relatively hyperbolic, relative to the peripheral subgroups, which are the stabilizers of individual horoballs. (Typically the list of peripheral subgroups is given by choosing one such stabilizer from each conjugacy class.) 
In $[\mathbf{2 5}]$ and [39], group-theoretic analogues of the $2 \pi$ theorem were proved. These theorems roughly state that if one begins with a relatively hyperbolic group, and kills off normal subgroups of the peripheral subgroups which contain no "short" elements, then the resulting group is also relatively hyperbolic, and "close" to the original group in various ways. From this one recovers a weaker group-theoretic version of the $2 \pi$ theorem, as the fundamental group of a hyperbolic 3-manifold is relatively hyperbolic, relative to the cusp groups; the operation of Dehn filling acts on fundamental groups by killing the cyclic subgroups generated by the filling slopes.

In the context of the original $2 \pi$ theorem, we begin with a group which has such an action on $\mathbb{H}^{3}$, which is not only $\delta$-hyperbolic, but which is actually $\operatorname{CAT}(-1)$. (See Section 3 for the definition of $\operatorname{CAT}(\kappa)$ for $\kappa \in \mathbb{R}$. For now, just note that $\mathrm{CAT}(-1)$ implies Gromov hyperbolic.) Moreover, the group acts cocompactly on a "neutered" $\mathbb{H}^{3}$, which is $\operatorname{CAT}(0)$ with isolated flats. The fundamental group of the filled manifold acts properly and cocompactly on a CAT $(-1) 3$-manifold.

For $\kappa \leq 0$, we say that a group is $C A T(\kappa)$ if it acts isometrically, properly, and cocompactly on some $\operatorname{CAT}(\kappa)$ space. We will say that a group is relatively $C A T(-1)$ if it has a geometrically finite action on a $\operatorname{CAT}(-1)$ space. A group is $C A T(0)$ with isolated flats if it acts isometrically, properly, and cocompactly on some CAT(0) space with isolated flats. (It follows from a result of Hruska and Kleiner [29] that horoballs can always be added to such a space to make it $\delta$-hyperbolic for some $\delta$. In particular, a group which is $\operatorname{CAT}(0)$ with isolated flats is relatively hyperbolic.) It is natural to wonder to what extent the main results of $[\mathbf{2 5}]$ and $[\mathbf{3 9}]$ can be strengthened in the presence of these stronger conditions.

Question 1.1. If one performs relatively hyperbolic Dehn filling on a relatively $C A T(-1)$ group, is the filled group always relatively $C A T(-1)$ ?

Question 1.2. If one performs relatively hyperbolic Dehn filling on a CAT(0) with isolated flats group, is the filled group always CAT(0) with isolated flats?

These questions form a part of the motivation for this paper; we answer them positively in some special cases. The utility of these answers is illustrated by using the additional CAT(0) structure of the quotient groups to obtain information about the filled groups which is less accessible from the coarse geometric viewpoint.

1.1. Outline. In Section 2, we describe the main results. In Section 3 , we recall the definition of warped product metric and an important result of Alexander and Bishop. We also discuss the space of directions and the logarithm map from a CAT(0) space to the tangent cone at 
a point. In Section 4, we extend original construction of the $2 \pi$ theorem to our setting, using doubly warped product constructions to give locally CAT(0) and locally CAT $(-1)$ models for our fillings. Section 5 contains the "Morse theoretic" arguments which give information about the boundary at infinity of the fundamental groups of our fillings. In Section 6, we pose some questions.

1.2. Acknowledgments. Fujiwara started working on this project during his visit at Max Planck Institute in Bonn in the fall of 2005. He has benefited from conversation with Potyagailo. The joint project with Manning started when he visited Caltech in April 2006. He thanks both institutions for their hospitality.

Manning thanks Ric Ancel for explaining how to prove Claim 5.22 in the proof of Proposition 5.20, and thanks Noel Brady and Daniel Groves for useful conversations.

\section{Statements of results}

2.1. Cones and fillings. We first establish some notation:

Definition 2.1 (Partial cone). Let $N$ be a flat torus of dimension $n$, and let $T$ be a totally geodesic $k$-dimensional submanifold in $N$, where $1 \leq k \leq n$. The torus $N$ has Euclidean universal cover $\mathbb{E}^{n} \rightarrow N$; let $\tilde{T} \cong \mathbb{E}^{k}$ be a component of the preimage of $T$ in $N$. The parallel copies of $\tilde{T}$ are the leaves of a fibration $\mathbb{E}^{n} \stackrel{\tilde{\pi}}{\longrightarrow} \mathbb{E}^{n-k}$. This fibration covers a fibration

$$
N \stackrel{\pi}{\longrightarrow} B
$$

of $N$ over some $(n-k)$-torus $B$. We define the partial cone $C(N, T)$ to be the mapping cylinder of $\pi$, i.e.

$$
C(N, T)=N \times[0,1] / \sim,
$$

where $\left(t_{1}, 1\right) \sim\left(t_{2}, 1\right)$ if $\pi\left(t_{1}\right)=\pi\left(t_{2}\right)$.

We refer to $N \times 0$ as the boundary of $C(N, T)$, and to $V(N, T):=$ $N \times 1 / \sim$ as the core.

Remark 2.2. The space $C(N, N)$ is the cone on $N$. In the case that $N$ is a metric product $N=T \times B$, then $C(N, T)$ can be canonically identified with $C(T) \times B$. (Although the identification is not canonical in general, $C(N, T)$ is always homeomorphic to $C(T) \times B$ for some torus $B$ of complementary dimension to that of $T$. In particular, the core $V(N, T)$ is always homeomorphic to a torus of $\operatorname{dimension} \operatorname{dim}(N)-$ $\operatorname{dim}(T)$.) The partial cone $C(N, T)$ is a manifold with boundary only when $\operatorname{dim}(T)=1$.

Definition 2.3. Let $M$ be a hyperbolic $(n+1)$-manifold. A toral cusp of $M$ is a neighborhood $E$ of an end of $M$ so that 


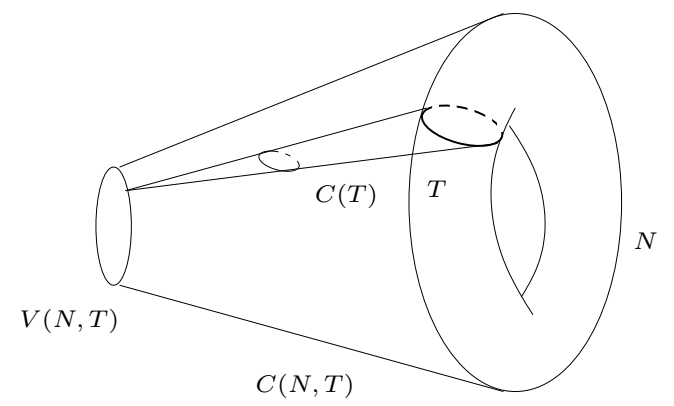

Figure 1. cone $C(N, T)$.

1) $E$ is homeomorphic to the product of a torus with the interval $(-\infty, 0]$, and

2 ) the induced Riemannian metric on $\partial E$ is flat.

For the rest of the section we make the following:

Standing assumption 2.4. $M$ is a hyperbolic $(n+1)$-manifold of finite volume containing disjoint toral cusps $E_{1}, \ldots, E_{m}$, and no other ends.

Let $\bar{M} \subset M$ be the manifold with boundary obtained by removing the interiors of the cusps. Let $N_{1}, \ldots, N_{m}$ be the components of $\partial \bar{M}$.

Definition 2.5 (Filling, $2 \pi$-filling). For each component $N_{i}$ of $\partial \bar{M}$ choose an embedded totally geodesic torus $T_{i}$ of dimension $k_{i}$. Form $C\left(N_{i}, T_{i}\right)$ as in the previous definition. We use the canonical identification $\phi_{i}$ of $\partial C\left(N_{i}, T_{i}\right)$ with $N_{i}$ to form the space

$$
M\left(T_{1}, \ldots, T_{m}\right):=\bar{M} \bigcup_{\phi_{1} \sqcup \cdots \sqcup \phi_{m}}\left(C\left(N_{1}, T_{1}\right) \sqcup \cdots \sqcup C\left(N_{m}, T_{m}\right)\right) .
$$

In this case, we say that $M\left(T_{1}, \ldots, T_{m}\right)$ is obtained from $M$ by filling along $\left\{T_{1}, \ldots, T_{m}\right\}$. We refer to the cores of the $C\left(N_{i}, T_{i}\right)$ as the filling cores.

If no $T_{i}$ contains a geodesic of length $2 \pi$ or less, we say $M\left(T_{1}, \ldots, T_{m}\right)$ is a $2 \pi$-filling of $M$.

Remark 2.6. The space $M\left(T_{1}, \ldots, T_{m}\right)$ described above is homeomorphic to a manifold if and only if every filling core has dimension exactly $n-1$. In any case $M\left(T_{1}, \ldots, T_{m}\right)$ is a pseudomanifold of dimension $(n+1)$, and the complement of the filling cores is homeomorphic to $M$.

On the level of fundamental groups, $\pi_{1}\left(M\left(T_{1}, \ldots, T_{m}\right)\right)$ is obtained from $\pi_{1}(M)$ by adjoining relations representing the generators of some direct summands of the cusp subgroups; namely, for each $i \in\{1, \ldots, m\}$, 
one adjoins relations corresponding to the generators of $\pi_{1}\left(T_{i}\right) \triangleleft \pi_{1}\left(E_{i}\right)=$ $\pi_{1}\left(N_{i}\right)$.

2.2. Main results. Our main theorem says that under hypotheses exactly analogous to those used in the $2 \pi$ theorem, a space obtained by filling as above can be given a nice locally CAT(0) metric; moreover, if all the filling cores are zero or one dimensional, the metric can be chosen to be locally $\mathrm{CAT}(-1)$. We remind the reader that, under the condition 2.4, $M$ is assumed to be a hyperbolic $(n+1)$-manifold with only toral cusps.

Theorem 2.7. Let $n \geq 2$ and assume the condition 2.4.

If $M\left(T_{1}, \ldots, T_{m}\right)$ is a $2 \pi$-filling of $M$, then there is a complete path metric d on $M\left(T_{1}, \ldots, T_{m}\right)$ satisfying the following:

1) The path metric $d$ is the completion of a path metric induced by a negatively curved Riemannian metric on the complement of the filling cores.

2) The path metric $d$ is locally $C A T(0)$.

3) If for every $i, T_{i}$ has codimension at most 1 in $\partial E_{i}$, then $d$ is locally $C A T(-\kappa)$ for some $\kappa>0$.

The metric constructed in Theorem 2.7 is identical to the original hyperbolic metric, away from a neighborhood of the filling cores. We will prove the following in the course of showing Theorem 2.7.

Proposition 2.8. Let $n \geq 2$ and assume the condition 2.4, and let $M\left(T_{1}, \ldots, T_{m}\right)$ be a $2 \pi$-filling of $M$. Let

$$
\bar{M}=M \backslash \bigcup_{i=1}^{m} E_{i}
$$

be as described in Definition 2.5. Choose $\lambda>0$ so that the $\lambda$-neighborhood of $\bigcup_{i=1}^{m} E_{i}$ is embedded in $M$. Let $\bar{M}^{\prime}$ be the complement in $M$ of the $\frac{\lambda}{2}$-neighborhood of $\bigcup_{i=1}^{m} E_{i}$. We thus have inclusions $\bar{M}^{\prime} \subset \bar{M} \subset M$, and $\bar{M}^{\prime} \subset \bar{M} \subset M\left(T_{1}, \ldots, T_{m}\right)$.

The Riemannian metric described in Theorem 2.7 is equal to the hyperbolic metric, when restricted to $\bar{M}^{\prime}$.

Remark 2.9. Let $G=\pi_{1}\left(M\left(T_{1}, \ldots, T_{m}\right)\right)$ be the fundamental group of some $2 \pi$-filling of $M$. It follows from Theorem 2.7 that $H_{n+1}(G) \cong$ $H_{n+1}\left(M\left(T_{1}, \ldots, T_{m}\right)\right)$ is cyclic. One can ask what the Gromov norm of a generator is, and define this number to be the simplicial volume of $M\left(T_{1}, \ldots, T_{m}\right)$ (or of $G$ ). In case $M\left(T_{1}, \ldots, T_{m}\right)$ is a manifold, this definition is the same as Gromov's in [21]. In the setting of the original $2 \pi$ theorem, both $M$ and $M\left(T_{1}, \ldots, T_{m}\right)$ are hyperbolic manifolds, and it was shown by Thurston that the simplicial volume of $M\left(T_{1}, \ldots, T_{m}\right)$ is bounded above by the hyperbolic volume of $M$, divided by a constant [41]. We extend Thurston's result to higher dimensions in [19]. 
Remark 2.10. Special cases of Theorem 2.7 appear in

- the Gromov-Thurston $2 \pi$-theorem $[\mathbf{2 0}, \mathbf{1 2}]$, in which $n=2$ and each filling core is a circle,

- Schroeder's generalization of the $2 \pi$ theorem to $n>2$, with filling cores equal to $(n-1)$-dimensional tori [40] (This is the case in which every $T_{i}$ is one-dimensional, and the only case in which the filling $M\left(T_{1}, \ldots, T_{m}\right)$ is a manifold.), and

- Mosher and Sageev's construction in [37] of $(n+1)$-dimensional hyperbolic groups which are not aspherical manifold groups; here the filling cores are points.

The construction of Mosher and Sageev is based on a discussion in [23, 7.A.VI], and the examples constructed by Mosher and Sageev answered a question which appeared in an early version of Bestvina's problem list [9]. The new examples of the current paper fall in some sense between those of Schroeder, and those of Mosher-Sageev.

Our construction gives the following corollary, which can be deduced from Theorem 2.7 and Proposition 4.21.

Corollary 2.11. Let $n \geq 2$ and assume the condition 2.4.

Let $M\left(T_{1}, \ldots, T_{m}\right)$ be a $2 \pi$-filling of $M$, and let $G$ be the fundamental group of $M\left(T_{1}, \ldots, T_{m}\right)$. Then $G$ is $C A T(0)$ with isolated flats. If each $T_{i}$ has dimension $n$ or $n-1$, then $G$ is $C A T(-1)$.

Thus Theorem 2.7 says that given a cusped hyperbolic $(n+1)-$ manifold with $n \geq 2$, there are many ways to fill its cusps to get a space whose universal cover is $\operatorname{CAT}(-\kappa)$ for $\kappa>0$. If $n \geq 3$, there are many ways to fill its cusps to get a space whose universal cover is CAT $(0)$ with isolated flats. The following proposition says that different fillings generally have different fundamental groups:

Proposition 2.12. Let $n \geq 2$ and assume the condition 2.4.

For each $i \in \mathbb{N}$ choose a filling $M_{i}=M\left(T_{1}^{i}, \ldots, T_{m}^{i}\right)$ of $M$, and suppose that the fillings $M_{i}$ satisfy: For each $1 \leq j \leq m$ and each element $\gamma$ of $\pi_{1}\left(E_{j}\right) \backslash\{1\}$, the set $\left\{i \mid \gamma \in \pi_{1}\left(T_{j}^{i}\right)\right\}$ is finite. Then for any $k$, there are only finitely many $i \in \mathbb{N}$ so that $\pi_{1}\left(M_{i}\right) \cong \pi_{1}\left(M_{k}\right)$.

Proof. We argue by contradiction. The hypotheses imply that all but finitely many fillings $M_{i}$ are $2 \pi$-fillings. By passing to a subsequence we may suppose that all the $\pi_{1}\left(M_{i}\right)$ are isomorphic to some fixed $G$. Applying Corollary 2.11 and [29, Theorem 1.2.1], $G$ is relatively hyperbolic with respect to a collection of free abelian subgroups. If $\Gamma=\pi_{1}(M)$ we have a sequence of surjections

$$
\Gamma \stackrel{\phi_{i}}{\longrightarrow} G
$$

obtained by killing subgroups $K_{j}^{i}$ of the peripheral subgroups of $\Gamma$ corresponding to the tori $T_{j}^{i}$. Since the injectivity radii of these tori are 
going to infinity, the length (with respect to a fixed generating set for $\Gamma$ ) of a shortest nontrivial element of $\cup_{j} K_{j}^{i}$ is also going to infinity. It follows from Corollary 9.7 of [25] that the stable kernel

$$
\underset{\operatorname{ker}}{\longrightarrow}\left\{\phi_{i}\right\}=\left\{\gamma \in \Gamma \mid \phi_{i}(\gamma)=1 \text { for almost all } \phi_{i}\right\}
$$

is trivial. Work of Groves then shows that $\Gamma$ is either abelian or splits over an abelian group [24, Proposition 2.11]. But $\Gamma$ is the fundamental group of a finite volume hyperbolic manifold of dimension at least 3 , so it cannot split over a virtually abelian subgroup (see, e.g. [6, Theorem 1.3.(i)]).

q.e.d.

We will show (Corollary 5.24) that no $2 \pi$-filling of an $(n+1)$-dimensional hyperbolic manifold with filling cores of dimension $<n$ has the same fundamental group as a closed hyperbolic manifold (or even a closed aspherical manifold) for $n \geq 3$. This is in sharp contrast to the situation for hyperbolic 3-manifolds. Combining Corollary 5.24 and Proposition 2.12 immediately yields the following:

Theorem 2.13. Let $n \geq 3$ and assume the condition 2.4. There are infinitely many $2 \pi$-fillings of $M$ whose fundamental groups are nonpairwise-isomorphic, torsion-free, $(n+1)$-dimensional hyperbolic groups, each of which acts geometrically on some $(n+1)$-dimensional $C A T(-1)$ space, and none of which is a closed aspherical manifold group.

If $n \geq 4$, there are infinitely many $2 \pi$-fillings of $M$ whose fundamental groups are non-pairwise-isomorphic, torsion-free, $(n+1)$ dimensional relatively hyperbolic groups, each of which acts geometrically on some $(n+1)$-dimensional CAT(0) space with isolated flats, and none of which is a closed aspherical manifold group. Flats of any dimension up to $(n-2)$ occur in infinitely many such fillings.

Januszkiewicz and Świạtkowski in [32] (and independently Haglund in [26]) introduce systolic groups, in part as a means of giving examples of non-manifold $n$-dimensional hyperbolic groups for all $n$ (see $[\mathbf{1 5}, \mathbf{3 1}]$ for other approaches). Recently, Osajda has shown that systolic groups are never simply connected at infinity [38]. It follows from our analysis of the group boundary in Section 5 that the high-dimensional groups obtained in Theorem 2.13 are simply connected at infinity whenever no filling cores are points. In particular, these groups are not systolic (Corollary 5.27).

\section{Preliminaries}

For general results and terminology for $\operatorname{CAT}(\kappa)$ spaces we refer to Bridson and Haefliger [13]. For convenience, we remind the reader of the definition. Let $\kappa \in \mathbb{R}$, and let $S_{\kappa}$ be the complete simply connected surface of constant curvature $\kappa$. Let $R_{\kappa} \leq \infty$ be the diameter of $S_{\kappa}$. 
Now let $X$ be a length space. (See $[\mathbf{1 3}, \mathbf{1 4}]$ for more on length spaces.) If $\Delta \subset X$ is a geodesic triangle in $X$ whose perimeter is bounded above by $2 R_{\kappa}$, with corners $x, y$, and $z$, then there is a comparison triangle $\bar{\Delta} \subset S_{\kappa}$; i.e., the corners of $\bar{\Delta}$ can be labelled $\bar{x}, \bar{y}$, and $\bar{z}$, and there is a map from $\Delta$ to $\bar{\Delta}$ which is an isometry restricted to any edge of $\Delta$, taking $x$ to $\bar{x}$, and so on (See Figure 2). If this map never decreases

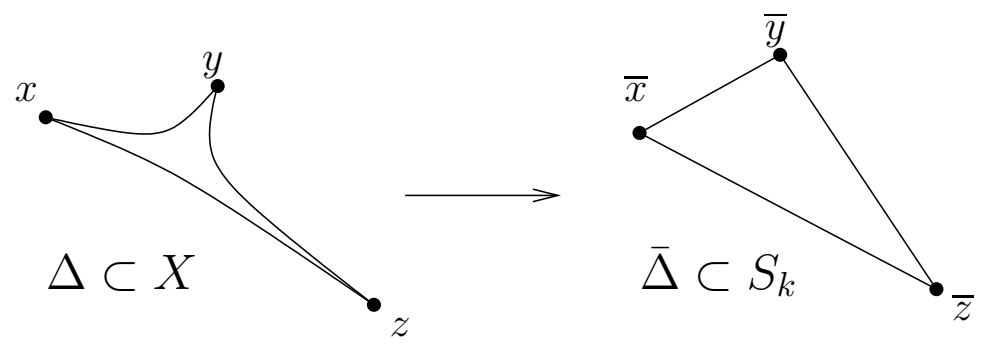

Figure 2. Comparison for $\operatorname{CAT}(k)$.

distance on any triangle $\Delta$ whose perimeter is bounded above by $2 R_{\kappa}$, we say that $X$ is $C A T(\kappa)$. Put informally, $X$ is $\operatorname{CAT}(\kappa)$ if its triangles are "no fatter" than the comparison triangles in $S_{\kappa}$. If every point in $X$ has a neighborhood which is $\operatorname{CAT}(\kappa)$, then we say that $X$ is locally $C A T(\kappa)$. If $X$ is locally $\operatorname{CAT}(0)$, then we say that $X$ is nonpositively curved. For $\kappa \leq 0$, the universal cover of a complete, locally $\operatorname{CAT}(\kappa)$ space is $\operatorname{CAT}(\kappa)$, by the Cartan-Hadamard Theorem.

3.1. Warped products. We take this definition from $[\mathbf{1 4}, 3.6 .4]$.

Definition 3.1. Given complete length spaces $\left(X, d_{X}\right)$ and $\left(Y, d_{Y}\right)$, and a continuous nonnegative $f: X \rightarrow[0, \infty)$, we can define the warped product of $X$ with $Y$, with warping function $f$ to be the length space based on the following method of measuring lengths of paths. Given any closed interval $[a, b]$ and any lipschitz path $\gamma: I \rightarrow X \times Y$, with $\gamma(t)=\left(\gamma_{1}(t), \gamma_{2}(t)\right)$, the quantities $\left|\gamma_{1}^{\prime}(t)\right|$ and $\left|\gamma_{2}^{\prime}(t)\right|$ are well defined almost everywhere on $I$ (see $[\mathbf{1 4}, 2.7 .6]$ ). Define the length of $\gamma, l(\gamma)$ to be

$$
l(\gamma)=\int_{a}^{b} \sqrt{\left|\gamma_{1}^{\prime}(t)\right|^{2}+f^{2}\left(\gamma_{1}(t)\right)\left|\gamma_{2}^{\prime}(t)\right|^{2}} d t .
$$

The quotient map $X \times Y \rightarrow X \times{ }_{f} Y$ is injective exactly when $f$ has no zeroes. If $f(x)=0$ then $\{x\} \times Y$ is identified to a point in $X \times_{f} Y$.

Often we will have $X \subseteq \mathbb{R}$, or $X=I \times{ }_{g} Z$ for $I \subseteq \mathbb{R}$, and $f$ depending only on $r \in I$. In this case, we will usually write $X \times_{f} Y$ as $X \times_{f(r)} Y$. For example:

- The euclidean plane is isometric to $[0, \infty) \times{ }_{r} S^{1}$. The subset $\{R\} \times$ $S^{1}$ is a circle of radius $R$. 
- The hyperbolic space $\mathbb{H}^{n+1}$ is isometric to $\mathbb{R} \times e_{e^{r}} \mathbb{E}^{n}$. The subset $\{R\} \times \mathbb{E}^{n}$ is a horosphere.

We will often abuse notation by using points in $X \times Y$ to refer to points in $X \times_{f} Y$, even when the quotient map is not injective. For example the cone point of the Euclidean cone $[0, \infty) \times_{r} X$ might be referred to as $(0, x)$ for some $x \in X$, or just as $(0,-)$.

In case the metrics $d_{X}$ and $d_{Y}$ are induced by Riemannian metrics $g_{X}$ and $g_{Y}$ and $f: X \rightarrow \mathbb{R}_{>0}$ is smooth, the warped product metric on $X \times{ }_{f} Y$ is equal to the path metric induced by a Riemannian metric $g$, given at a point $(x, y)$ by the formula

$$
g=g_{X}+f^{2}(x) g_{Y} .
$$

Curvature bounds on warped product spaces are studied by Alexander and Bishop in [4], where quite general sufficient conditions are given for a warped product to have curvature bounded from above or below. In order to state the results from [4] which we need, we first recall some definitions.

Definition 3.2. Let $K \in \mathbb{R}$. Let $\mathcal{F} K$ be the set of solutions to the differential equation $g^{\prime \prime}+K g=0$. A function $f$ satisfying $f \leq g$ for any function $g$ in $\mathcal{F} K$ agreeing with $f$ at two points which are sufficiently close together is said to satisfy the differential inequality $f^{\prime \prime}+K f \geq 0$ in the barrier sense.

Definition 3.3. Let $K \in \mathbb{R}$, and let $B$ be a geodesic space. A continuous function $f: B \rightarrow \mathbb{R}$ is $\mathcal{F} K$-convex if its composition with any unit-speed geodesic satisfies the differential inequality $f^{\prime \prime}+K f \geq 0$ in the barrier sense.

We collect here some useful facts about $\mathcal{F} K$-convexity:

\section{Useful facts 3.4.}

1) A function is $\mathcal{F} 0$-convex if and only if it is convex in the usual sense.

2) A non-negative $\mathcal{F} K$-convex function is also $\mathcal{F} K^{\prime}$-convex for any $K^{\prime} \geq K$.

3) $[3$, Theorem 1.1(1A)] If $B$ is $\mathrm{CAT}(-1)$ and $x \in B$, then $\cosh (d(\cdot, x))$ is a $\mathcal{F}(-1)$-convex function on $B$.

4) $[\mathbf{3}$, Theorem $1.1(3 \mathrm{~A})]$ If $A$ is a convex subset of a $\mathrm{CAT}(0)$ space $B$, then $d(\cdot, A)$ is a convex function on $B$.

5) $[3$, Theorem $1.1(4 \mathrm{~A})]$ If $A$ is a convex subset of a $\mathrm{CAT}(-1)$ space $B$, then $\sinh (d(\cdot, A))$ is a $\mathcal{F}(-1)$-convex function on $B$.

We now state a special case of a more general result in [4].

Theorem 3.5. [4, Theorem 1.1] Let $K \leq 0$, and let $K_{F} \in \mathbb{R}$. Let $B$ and $F$ be complete locally compact $C A T(K)$ and $C A T\left(K_{F}\right)$ spaces, respectively. Let $f: B \rightarrow[0, \infty)$ be $\mathcal{F} K$-convex. Let $X=f^{-1}(0)$. 
Suppose further that either

1) $X=\emptyset$ and $K_{F} \leq K(\inf f)^{2}$, or

2) $X$ is nonempty and $(f \circ \sigma)^{\prime}\left(0^{+}\right)^{2} \geq K_{F}$ whenever $\sigma:[0, x] \rightarrow B$ is a shortest (among all paths starting in $X$ ) unit-speed geodesic from $X$ to a point $b \in B \backslash X$.

Then the warped product $B \times{ }_{f} F$ is $C A T(K)$.

3.2. The space of directions and the tangent cone. Suppose that $X$ is a length space, and $p \in X$. Recall that the space of directions at $p$ is the collection of geodesic segments issuing from $p$, modulo the equivalence relation: $[p, x] \sim[p, y]$ if the Alexandroff angle between $[p, x]$ and $[p, y]$ at $p$ is zero. The Alexandroff angle at $p$ makes the space of directions, $\Sigma_{p}(X)$, into a metric space. If $X$ is a Riemannian manifold, then $\Sigma_{p}(X)$ is always a round sphere of diameter $\pi$. The tangent cone at $p$ is the Euclidean cone on the space of directions at $p$ :

$$
C_{p}(X) \cong[0, \infty) \times_{r} \Sigma_{p}(X) .
$$

We will sometimes just write $\Sigma_{p}$ and $C_{p}$ if the space $X$ is understood. If $X$ is a Riemannian manifold near the point $p$, then the tangent cone $C_{p}$ can be canonically identified with the tangent space of $X$ at $p$.

In our setting $X$ will always be geodesically complete and locally compact. With these assumptions, if $U$ is any open neighborhood of $p \in X$, then $C_{p}(X)$ is isometric to the pointed Gromov-Hausdorff limit of spaces $U_{n}$ isometric to $U$ with the metric multiplied by factors $R_{n}$ going to infinity (see for example [14, Theorem 9.1.48]).

Alexander and Bishop [4, page 1147] observe that the space of directions at a point in a warped product is determined by the spaces of directions in the factors together with infinitesimal information about the warping functions. If $f: B \rightarrow \mathbb{R}$ is a convex function and $p \in \mathbb{R}$, then $f$ has a well-defined "derivative" $D f_{p}: C_{p}(B) \rightarrow \mathbb{R}$ at the point $p$; this derivative is linear on each ray.

Definition 3.6 (spherical join, cf. $[\mathbf{1 3}, \mathrm{I} .5]$ ). Let $A$ and $B$ be metric spaces. We may give the product $\left[0, \frac{\pi}{2}\right] \times A \times B$ a pseudometric as follows. Let $x_{1}=\left(\phi_{1}, a_{1}, b_{1}\right)$ and $x_{2}=\left(\phi_{2}, a_{2}, b_{2}\right)$. The distance $d\left(x_{1}, x_{2}\right)$ is given by

$$
\begin{aligned}
\cos \left(d\left(x_{1}, x_{2}\right)\right)= & \cos \left(\phi_{1}\right) \cos \left(\phi_{2}\right) \cos \left(d_{\pi}\left(a_{1}, a_{2}\right)\right) \\
& +\sin \left(\phi_{1}\right) \sin \left(\phi_{2}\right) \cos \left(d_{\pi}\left(b_{1}, b_{2}\right)\right),
\end{aligned}
$$

where $d_{\pi}\left(p_{1}, p_{2}\right)$ means $\min \left\{\pi, d\left(p_{1}, p_{2}\right)\right\}$. The spherical join $A * B$ of $A$ and $B$ is the canonical metric space quotient of $\left[0, \frac{\pi}{2}\right] \times A \times B$ with the above metric. Points in $A * B$ can be written as triples $(\phi, a, b)$ with $\phi \in\left[0, \frac{\pi}{2}\right], a \in A$ and $b \in B$. If $\phi=0$, the third coordinate can be ignored, and if $\phi=\frac{\pi}{2}$, the second coordinate can be ignored. 
Proposition 3.7. [4] Let B, F, and $f$ be as in Theorem 3.5. Let $p \in B$, and let $\phi \in F$. If $f(p)>0$, then there are isometries

$$
C_{(p, \phi)}\left(B \times_{f} F\right) \cong C_{p}(B) \times C_{\phi}(F)
$$

and

$$
\Sigma_{(p, \phi)}\left(B \times_{f} F\right) \cong \Sigma_{p}(B) * \Sigma_{\phi}(F) .
$$

If $f(p)=0$, there are isometries

$$
C_{(p, \phi)}\left(B \times_{f} F\right) \cong C_{p}(B) \times_{D f_{p}} F
$$

and

$$
\Sigma_{(p, \phi)}\left(B \times_{f} F\right) \cong \Sigma_{p}(B) \times_{D f_{p}} F
$$

3.3. Locally injective logarithms. Let $X$ be a uniquely geodesic metric space and let $p \in X$. In general, there is no exponential map defined on the entire tangent cone $C_{p}$. However, there is a well-defined logarithm map from $X$ to $C_{p}[\mathbf{3 3}]$.

Definition 3.8. Let $X$ be a uniquely geodesic metric space, and let $C_{p}=[0, \infty) \times_{r} \Sigma_{p}$ be the tangent cone at a point $p \in X$. If $x \in X$, then $\log _{p}(x)$ is the equivalence class of $(d(x, p),[p, x])$ in $C_{p}$.

If the metric is only locally uniquely geodesic, then the logarithm map can still be defined in a neighborhood of each point. The existence of a local exponential map is limited by the extent to which the logarithm is injective and surjective.

Definition 3.9. The $\log$-injectivity radius at $p, \operatorname{loginj}(p)$, is be the supremum of those numbers $r$ for which $\log _{p}$ is well-defined and injective on the ball of radius $r$ about $p$. A neighborhood of $p$ on which $\log _{p}$ is injective is called a log-injective neighborhood of $p$.

Similarly, the log-surjectivity radius at $p, \log \operatorname{surj}(p)$, is the supremum of those numbers $r$ for which $[0, r] \times{ }_{r} \Sigma_{p} \subset C_{p}$ is contained in the image of $\log _{p}$.

In general, neither $\operatorname{loginj}(p)$ nor $\operatorname{logsurj}(p)$ is positive. Recall that a space has the geodesic extension property if any locally geodesic segment terminating at $y$ can be extended to a longer locally geodesic segment terminating at some $y^{\prime} \neq y$. A complete $\operatorname{CAT}(0)$ space satisfying the geodesic extension property has $\log \operatorname{surj}(p)=\infty$.

Observation 3.10. If $R=\min \{\operatorname{logsurj}(p), \operatorname{loginj}(p)\}$, then there is an exponential map

$$
\exp _{p}:[0, R) \times_{r} \Sigma_{p} \rightarrow X
$$

which is a homeomorphism onto the open $R$-neighborhood of $p$. More generally there is always an exponential map

$$
\exp _{p}: \log _{p}(U) \rightarrow U \subset X
$$


for any $\log$-injective neighborhood $U$ of $p$, and this map is a homeomorphism onto its image.

Definition 3.11. A subset $V$ of a uniquely geodesic space is convex if for any two points in $V$, the geodesic connecting them is contained in $V$. The subset $V$ is strictly convex if it is convex and if the frontier of $V$ contains no non-degenerate geodesic segment.

Lemma 3.12. Let $X$ be a proper uniquely geodesic space satisfying the geodesic extension property. Suppose that $C$ is a compact strictly convex set in $X$ containing $p$ in its interior, and suppose that $C$ is contained in a log-injective neighborhood of $p$. It follows that the frontier of $C$ is homeomorphic to $\Sigma_{p}$.

Proof. Since under the hypotheses both the space of directions $\Sigma_{p}$ and the frontier of $C$ (which we denote here by $\partial C$ ) are compact Hausdorff spaces, it suffices to find a continuous bijection from one to the other.

Let $h: \partial C \rightarrow \Sigma_{p}$ be the map which sends a point $x$ to the equivalence class of $[x, p]$. Since $p \notin \partial C$, this map is well-defined. Since $X$ is a proper uniquely geodesic space, geodesic segments vary continuously with their endpoints [13, I.3.13]. It follows that the map $h$ is continuous.

We next show that $h$ is surjective. Let $\theta \in \Sigma_{p}$, and choose a geodesic segment beginning at $p$ with direction $\theta$. Since $X$ satisfies the geodesic extension property, this geodesic segment can be extended to a point $x$ not in $C$. Some point on the geodesic $[p, x]$ must be in the frontier of $C$, and so $\theta$ is in the image of $C$.

Finally we show that $h$ is injective. Choose $\theta \in \Sigma_{p}$, and suppose that $h(x)=h(y)=\theta$ for some $x, y$ in $\partial C$. Without loss of generality, suppose that $d(x, p) \leq d(y, p)$. Since $C$ is strictly convex, the geodesic from $x$ to $y$ must be contained in $C$ but cannot be contained entirely in $\partial C$. Thus there is some point $z \in[x, y]$ so that $z$ is in the interior of $C$. By the hypotheses and Observation 3.10 there is an open set $U \subset C_{p}=$ $[0, \infty) \times_{r} \Sigma_{p}$ containing $\log _{p}(C)$ so that the exponential map $\exp _{p}$ is a homeomorphism from $U$ to its image. The point $\log _{p}(z)$ is contained in some basic open subset of $U$ of the form $(d(z, p)-\epsilon, d(z, p)+\epsilon) \times V$ where $V$ is an open set in $\Sigma_{p}$ containing $\theta$. But since $C$ is convex, $\log _{p}(C)$ must also contain the union of the segments beginning at $p$ and ending in $(d(z, p)-\epsilon, d(z, p)+\epsilon) \times V$; in other words, $\log _{p}(C)$ must contain $(0, d(z, p)+\epsilon) \times V$. In particular, $C$ contains an open neighborhood of any point on the geodesic between $p$ and $z$, and thus $x$ must be an interior point of $C$, which is a contradiction.

q.e.d.

\section{The metric construction and proof of Theorem 2.7}

In this section we prove Theorem 2.7 by constructing suitable warped product metrics on the "partial cones" $C\left(N_{i}, T_{i}\right)$ (see Definition 2.1) so 
that those metrics are compatible with the hyperbolic metric on $\bar{M}$. The metric will be shown to be CAT(0) by applying Theorem 3.5 near the singular part, and by computing the sectional curvatures in the Riemannian part.

4.1. A model for the singular part. We first make an observation about warped products, whose proof we leave to the reader.

Lemma 4.1. Let $I \subseteq \mathbb{R}$ be connected, and let $f: I \rightarrow[0, \infty)$, and let $F$ be a geodesic metric space. Suppose that $f$ has a local minimum at $z \in I$.

1) $\{z\} \times F$ is a convex subset of $Z=I \times{ }_{f} F$.

2) Let $y \in I$, and $x \in F$. The shortest path from $(y, x)$ to $z \times F$ in $Z$ is $[y, z] \times\{x\}$.

We next give some applications of Theorem 3.5.

Lemma 4.2. Let $E$ be complete and $C A T(k)$ for $k \in[-1,0]$. The warped product

$$
B=[0, \infty) \times \cosh (r) E
$$

is $C A T(k)$, and $\{(0, e) \mid e \in E\} \subset B$ is convex.

Proof. Since $\cosh (r)$ is $\mathcal{F}(-1)$ convex, it is $\mathcal{F}(k)$-convex, by 3.4.(2). The zero set $\cosh ^{-1}(0)$ is empty, and $\inf _{r} \cosh (r)=1$, so condition (1) of Theorem 3.5 is verified with $K=K_{F}=k$. It follows that $B$ is $\operatorname{CAT}(k)$. Since the warping function $\cosh (r)$ has a minimum at 0 , Lemma 4.1 implies that $\{0\} \times E$ is convex.

q.e.d.

Proposition 4.3. Suppose that $E$ is complete and $C A T(k)$ for $k \in$ $\{-1,0\}$, and suppose that $F$ is $C A T(1)$. The space

$$
W=[0, \infty) \times \cosh (r) E \times \sinh (r) F
$$

is $C A T(k)$, and $\{(0, e,-) \mid e \in E\} \subset W$ is convex.

Proof. By Lemma 4.1, the set $Y=\{0\} \times E$ is a convex subset of $B=[0, \infty) \times_{\cosh (r)} E$. On $B$, the function $d(r, e)=r$ is the distance to $Y$. By Lemma 4.2 and Useful fact 3.4.(4), the function $d(\cdot, Y)$ is convex on $B$

Suppose $k=0$. The function $\sinh (r)$ is the composition of an increasing convex function with a convex function, hence it is convex. If $k=-1$, then $\sinh (r)$ is $\mathcal{F}(-1)$-convex by 3.4.(5). In either case, $\sinh (r)$ is $\mathcal{F} k$-convex, and so we can try to apply Theorem 3.5 to the warped product

$$
W=B \times \sinh (r) F .
$$

We must verify condition (2) of Theorem 3.5 , since $Y=\sinh ^{-1}(0)$ is nonempty. Let $b=(z, e) \in B \backslash Y$. We can apply the second part of Lemma 4.1 to see that a shortest unit speed geodesic $\sigma$ from $F$ to $b$ is of 
the form $\sigma(t)=(t, e)$, with domain $[0, z]$. We thus have $(\sinh \circ \sigma)^{\prime}\left(0^{+}\right)=$ $\cosh (0)=1 \geq 1$ as required.

Finally, we show that $Z=\{(0, e,-) \mid e \in E\}$ is convex. If $\sigma:[0,1] \rightarrow$ $W$ is any rectifiable path with both endpoints in $Z$, we can write $\sigma$ in coordinates as $\sigma(t)=(r(t), e(t), \theta(t))$ and note that if $\sigma$ leaves $Z$, then $\bar{\sigma}(t)=(0, e(t),-)$ is a shorter path with the same endpoints. It follows that any geodesic with both endpoints in $Z$ must lie entirely in $Z$.

q.e.d.

Using the convention that $\mathbb{E}^{0}$ is a point, the euclidean space $\mathbb{E}^{k}$ is $\operatorname{CAT}(0)$ for all $n$, and is $\operatorname{CAT}(-1)$ for $k=0$ and $k=1$. We thus have:

Corollary 4.4. Let $T$ be a complete CAT(1) space, and let $k$ be a nonnegative integer. The warped product

$$
\tilde{F}=[0, \infty) \times{ }_{\cosh (r)} \mathbb{E}^{k} \times_{\sinh (r)} T
$$

is complete and $C A T(0)$. If $k \leq 1$, then $\tilde{F}$ is $C A T(-1)$.

Lemma 4.5. Let $T$ be a flat manifold. The warped product

$$
\tilde{F}=[0, \infty) \times \cosh (r) \mathbb{E}^{k} \times \sinh (r) T
$$

is the metric completion of the Riemannian manifold

$$
D=(0, \infty) \times \cosh (r) \mathbb{E}^{k} \times_{\sinh (r)} T .
$$

Proof. The manifold $D$ is clearly dense in $\tilde{F}$, so it suffices to show that $D$ includes isometrically in $\tilde{F}$. Let $x_{1}=\left(r_{1}, e_{1}, t_{1}\right)$ and $x_{2}=\left(r_{2}, e_{2}, t_{2}\right)$ be two points in $D$, and let $\epsilon>0$. Let $\gamma$ be a lipschitz path in the product $[0, \infty) \times \mathbb{E}^{k} \times T$ nearly realizing the distance between $x_{1}$ and $x_{2}$ in $\tilde{F}$, so that the length in $\tilde{F}$ of $\gamma$ is at most $d_{\tilde{F}}\left(x_{1}, x_{2}\right)+\epsilon$. We will replace $\gamma$ by a path $\gamma^{\prime}$ in $D$ so that the length of $\gamma^{\prime}$ exceeds the length of $\gamma$ by at most $\delta(\epsilon)$, with $\lim _{\epsilon \rightarrow 0^{+}} \delta(\epsilon)=0$. Letting $\epsilon$ tend to zero, the lemma will follow.

For $t$ in the domain of $\gamma$, we have $\gamma(t)=(r(t), e(t), \theta(t))$. If $r(t)$ is positive for all $t$, then $\gamma$ stays inside $D$, and there is nothing to prove. We therefore assume that $r(t)=0$ for $t$ in a (possibly degenerate) interval $\left[t_{1}, t_{2}\right]$. By Proposition $4.3,\left\{(0, e,-) \mid e \in \mathbb{E}^{k}\right\}$ is convex, so we have $r(t)>0$ for any $t \notin\left[t_{1}, t_{2}\right]$.

The coordinates of $\gamma$ are uniformly continuous in $t$, so we can find small positive $\alpha_{1}$ and $\alpha_{2}$ so that $r\left(t_{1}-\alpha_{1}\right)=r\left(t_{2}+\alpha_{2}\right)$ and all the following are satisfied:

$$
\begin{aligned}
\max \left\{d\left(e\left(t_{1}-\alpha_{1}\right), e\left(t_{1}\right)\right), d\left(e\left(t_{2}+\alpha_{2}\right), e\left(t_{2}\right)\right)\right\} & <\epsilon \\
\max \left\{d\left(\theta\left(t_{1}-\alpha_{1}\right), \theta\left(t_{1}\right)\right), d\left(\theta\left(t_{2}+\alpha_{2}\right), \theta\left(t_{2}\right)\right)\right\} & <\epsilon \\
\max \left\{\cosh \left(r\left(t_{1}-\alpha_{1}\right)\right)-1, \sinh \left(r\left(t_{1}-\alpha_{1}\right)\right)\right\} & <\epsilon .
\end{aligned}
$$

Let $e^{\prime}:\left[t_{1}-\alpha_{1}, t_{2}+\alpha_{2}\right] \rightarrow \mathbb{E}^{k}$ be a constant-speed geodesic from $e\left(t_{1}-\right.$ $\left.\alpha_{1}\right)$ to $e\left(t_{2}+\alpha_{2}\right)$, and let $\theta^{\prime}:\left[t_{1}-\alpha_{1}, t_{2}+\alpha_{2}\right]$ be a constant-speed 
geodesic from $\theta\left(t_{1}-\alpha_{1}\right)$ to $\theta\left(t_{2}+\alpha_{2}\right)$. Let $\gamma^{\prime}$ be given by

$$
\gamma^{\prime}(t)= \begin{cases}\gamma(t) & t<t_{1}-\alpha_{1} \\ \left(r\left(t_{1}-\alpha_{1}\right), e^{\prime}(t), \theta^{\prime}(t)\right) & t_{1}-\alpha_{1} \leq t \leq t_{2}+\alpha_{2} \\ \gamma(t) & t>t_{2}+\alpha_{2}\end{cases}
$$

As the reader may check, the difference between the length of $\gamma$ and the length of $\gamma^{\prime}$ is at most $\epsilon\left(d\left(\theta\left(t_{1}\right), \theta\left(t_{2}\right)+d\left(e\left(t_{1}\right), e\left(t_{2}\right)\right)+\epsilon+2 \epsilon^{2}\right.\right.$. Letting $\epsilon$ tend to zero, we have established the lemma.

q.e.d.

If $T$ is a Riemannian manifold, the warped product from Corollary 4.4 is a Riemannian manifold in a neighborhood of any $p \notin\{0\} \times \mathbb{E}^{k} \times T$. It follows that the space of directions at $p$ is a sphere. The next lemma describes the space of directions at a non-manifold point.

Lemma 4.6. Let $\tilde{F}$ be as in Corollary 4.4. The space of directions $\Sigma_{(0, e,-)}(\tilde{F})$ at a point $(0, e,-)$ of $\tilde{F}$ is isometric to the spherical join $S^{k-1} * T$.

Proof. Proposition 3.7 can be applied twice, as follows. Let $B=$ $[0, \infty) \times \sinh (r) T$, and apply the second part of Proposition 3.7 to deduce

$$
\Sigma_{(0,-)}(B) \cong T \text {. }
$$

Next, since $\tilde{F} \cong B \times{ }_{\cosh r} \mathbb{E}^{k}$, we can apply the first part of Proposition 3.7 to deduce that

$$
\Sigma_{(0, e,-)}(\tilde{F}) \cong \Sigma_{(0,-)}(B) * \Sigma_{e}(E) .
$$

q.e.d.

Lemma 4.7. Suppose $T$ is a complete flat Riemannian manifold with injectivity radius bigger than $\pi$. Then $\tilde{F}=[0, \infty) \times_{\cosh (r)} \mathbb{E}^{k} \times_{\sinh (r)} T$ has the geodesic extension property.

Proof. Since $\tilde{F}$ is $\operatorname{CAT}(0)$ by Corollary 4.4 , geodesics are the same as local geodesics. Away from $V=\left\{(0, e,-) \mid e \in \mathbb{E}^{k}\right\}$, the space $\tilde{F}$ is Riemannian, so geodesics can be extended in $\tilde{F} \backslash V$.

At a point $p$ of $V$, Lemma 4.6 implies that the space of directions $\Sigma_{p}$ is isometric to $S^{k-1} * T$. Suppose $\sigma$ is a geodesic segment terminating at $p$, and let $[\sigma]=(\phi, \alpha, \theta) \in \Sigma_{p}$ be the direction of $\sigma$, where $\phi \in\left[0, \frac{\pi}{2}\right]$, $\alpha \in S^{k-1}$, and $\theta \in T$. Since $T$ has injectivity radius bigger than $\pi$, there is some $\theta^{\prime}$ with $d_{T}\left(\theta, \theta^{\prime}\right)=\pi$. It is straightforward to check that the Alexandrov angle between $[\sigma]$ and $\left(\phi,-\alpha, \theta^{\prime}\right)$ is $\pi$. Letting $\sigma^{\prime}$ be any geodesic segment starting at $p$, with direction $\left(\phi,-\alpha, \theta^{\prime}\right)$, we see that $\sigma^{\prime}$ geodesically extends $\sigma$ past $p$.

q.e.d.

We next argue that the space $\tilde{F}$ described in Corollary 4.4 has loginjective neighborhoods at the singular points, in the sense of Definition 3.9. It suffices to show that geodesic segments emanating from a point 
in the singular set can only make an angle of zero if they coincide on an initial subsegment. We first find the direction of an arbitrary geodesic segment emanating from a point in the singular set.

Lemma 4.8. Let $T$ be a flat manifold of injectivity radius larger than $\pi$. Let $p_{0}$ be a point in $E=\{0\} \times \mathbb{E}^{k} \subset[0, \infty) \times \cosh (r) \mathbb{E}^{k} \times_{\sinh (r)} T$, and let $p_{1}$ be some other point of $\tilde{F}$; that is, $p_{0}=\left(0, a_{0},-\right)$ and $p_{1}=\left(t_{1}, a_{1}, \theta_{1}\right)$ for some $t_{1} \geq 0, a_{0}$ and $a_{1} \in E$ and $\theta_{1} \in T$. Let $\sigma$ be a geodesic from $p_{0}$ to $p_{1}$. If $a_{1}=a_{0}$, then the direction of $\sigma$ at $p_{0}$ (in the spherical join coordinates of Definition 3.6) is $\left(\frac{\pi}{2},-, \theta_{1}\right)$. Otherwise let $\alpha$ be the direction of a geodesic in $E$ from $a_{0}$ to $a_{1}$; the direction of $\sigma$ is $\left(\phi, \alpha, \theta_{1}\right)$ where

$$
\tan (\phi)=\frac{\tanh \left(t_{1}\right)}{\sinh \left(\left|a_{1}-a_{0}\right|\right)}
$$

Proof. If $a_{1}=a_{0}$ the Lemma is obvious. Otherwise, we identify $\mathbb{R}$ with a geodesic $\gamma$ in $E$ passing through $a_{0}$ and $a_{1}$, setting $a_{0}=0$. The warped product $W=[0, \infty) \times_{u \cosh (t)} \mathbb{R} \subset \tilde{F}$ contains the geodesic from $p_{0}$ to $p_{1}$.

The map $h(t, a)=e^{u a}(\tanh t+i$ sech $t)$ takes $W$ isometrically onto the hyperbolic half plane in the upper half-space model consisting of points with nonnegative real part. The map $h$ takes $p_{0}$ to $i$ and $p_{1}$ to some point of modulus at least 1 . The geodesic $\gamma$ is sent to the positive imaginary axis. It is an exercise in hyperbolic geometry to verify that if the geodesic from $h\left(p_{0}\right)$ to $h\left(p_{1}\right)$ makes an angle of $\phi$ with $h(\gamma)$, then $\tan (\phi)=\frac{\tanh \left(t_{1}\right)}{\sinh \left(\left|a_{1}-a_{0}\right|\right)}$. The lemma follows. $\quad$ q.e.d.

Corollary 4.9. Let $p=(0, a,-)$ lie in $E \subset \tilde{F}$. Any open neighborhood of $p$ is log-injective at $p$.

Proof. Let $p_{1}=\left(t_{1}, a_{1}, \theta_{1}\right)$ and $p_{2}=\left(t_{2}, a_{2}, \theta_{2}\right)$ be points of $\tilde{F}=$ $[0, \infty) \times_{\cosh (r)} \mathbb{E}^{k} \times_{\sinh (r)} T$, and suppose that $\log \left(p_{1}\right)=\log \left(p_{2}\right)$ in the tangent cone at $p$. Let $\gamma_{i}$ be the unique geodesic from $p$ to $p_{i}$, for $i \in\{1,2\}$. Since $\log \left(p_{1}\right)=\log \left(p_{2}\right)$, the geodesic segments $\gamma_{1}$ and $\gamma_{2}$ have the same length and the same direction $(\phi, \alpha, \theta)$ as described in Lemma 4.8. In particular, the segments $\left[a, a_{1}\right]$ and $\left[a, a_{2}\right]$ in $E$ must have the same direction at $a$. The log-injectivity radius at any point in Euclidean space is infinite, so these geodesic segments are subsets of a single line in $E$. As in the proof of Lemma 4.8, the geodesics $\gamma_{1}$ and $\gamma_{2}$ both lie in a subset of $\tilde{F}$ isometric to a hyperbolic halfplane. Since a hyperbolic half-plane has infinite log-injectivity radius, $\gamma_{1}$ and $\gamma_{2}$ must coincide.

q.e.d.

Remark 4.10. If one is merely interested in nonpositive curvature, and not log-injectivity, there is more flexibility in the choice of warping functions. Suppose that $T$ is a flat manifold. It is not hard to show that 
the space

$$
\tilde{F}^{\prime}=[0, \infty) \times_{e^{r}} \mathbb{E}^{k} \times_{\sinh (r)} T
$$

is $\operatorname{CAT}(0)$. On the other hand, $\tilde{F}^{\prime}$ does not have log-injective neighborhoods at points $(0, e,-)$.

4.2. Curvatures in Riemannian warped products. The following proposition can be proved by a simple if tedious computation involving Christoffel symbols. See [12] for a proof in case both flat factors are 1 -dimensional.

Proposition 4.11. Let $A_{1}$ and $A_{2}$ be flat manifolds, and let $I \subseteq$ $\mathbb{R}$. Let $f_{1}, f_{2}$ be positive smooth functions on $I$. The warped product manifold

$$
W=I \times{ }_{f_{1}} A_{1} \times_{f_{2}} A_{2}
$$

has sectional curvatures at $\left(t, a_{1}, a_{2}\right) \in W$ which are convex combinations of the following functions, evaluated at $t$ :

$$
-\frac{f_{1}^{\prime \prime}}{f_{1}},-\frac{f_{2}^{\prime \prime}}{f_{2}},-\frac{\left(f_{1}^{\prime}\right)^{2}}{f_{1}^{2}},-\frac{\left(f_{2}^{\prime}\right)^{2}}{f_{2}^{2}}, \text { and }-\frac{f_{1}^{\prime} f_{2}^{\prime}}{f_{1} f_{2}} .
$$

Let $i \in\{1,2\}$. If $\operatorname{dim}\left(A_{i}\right)=1$, then the term $-\frac{\left(f_{i}^{\prime}\right)^{2}}{f_{i}^{2}}$ can be ignored. If $\operatorname{dim}\left(A_{i}\right)=0$, then all terms involving $f_{i}$ and its derivatives can be ignored.

It follows from this proposition that if $f_{1}$ and $f_{2}$ are positive, convex, and increasing, the warped product $I \times{ }_{f_{1}} A \times_{f_{2}} B$ is nonpositively curved. If $f_{1}, f_{2}$, and their first and second derivatives are all bounded between two positive numbers $\epsilon<1$ and $R>1$, then the curvature of $I \times{ }_{f_{1}} A \times_{f_{2}}$ $B$ is bounded between $-\frac{R^{2}}{\epsilon^{2}}$, and $-\frac{\epsilon^{2}}{R^{2}}$.

4.3. Gluing functions. We will construct smooth convex increasing functions $f$ and $g$ on $[0,1+\lambda]$ interpolating between the exponential function $e^{r-1}$ near $r=\lambda$, and the functions $\sinh (r)$ and $\cosh (r)$ near $r=0$. We will apply the following result of Agol, proved in [2].

Lemma 4.12. [2, Lemma 2.5] Suppose

$$
a(r)= \begin{cases}b(r) & r<R, \\ c(r) & r \geq R,\end{cases}
$$

where $b(r)$ and $c(r)$ are $C^{\infty}$ on $(-\infty, \infty)$, and $b(R)=c(R), b^{\prime}(R)=$ $c^{\prime}(R)$. Then we may find $C^{\infty}$ functions $a_{\epsilon}$ on $(-\infty, \infty)$ for $\epsilon>0$ such that

1) there is a $\delta(\epsilon)>0$ such that $\lim _{\epsilon \rightarrow 0^{+}} \delta(\epsilon)=0$ and $a_{\epsilon}(r)=b(r)$ for $r \leq R-\delta(e), a_{\epsilon}(r)=c(r)$ for $r \geq R$, and 
2)

$$
\begin{aligned}
& \min \left\{b^{\prime \prime}(R), c^{\prime \prime}(R)\right\}=\lim _{\epsilon \rightarrow 0^{+}} \inf _{-\delta(\epsilon) \leq r \leq R} a_{\epsilon}^{\prime \prime}(r) \\
\leq & \lim _{\epsilon \rightarrow 0^{+}} \sup _{R-\delta(\epsilon) \leq r \leq R} a_{\epsilon}^{\prime \prime}(r)=\max \left\{b^{\prime \prime}(R), c^{\prime \prime}(R)\right\} .
\end{aligned}
$$

Agol's lemma allows us to smoothly interpolate between functions which agree up to first order at some point. We wish to interpolate between pairs of functions (either $\sinh (r)$ and $e^{r-1}$ or $\cosh (r)$ and $e^{r-1}$ ) which do not agree anywhere up to first order. To solve this difficulty, we introduce a third function which interpolates between the pair:

Lemma 4.13. Let $l_{1}(x)=a x+b, l_{2}(x)=c x+d$. Suppose $a<c$, and let $[A, B]$ be an interval containing $\frac{b-d}{c-a}$ (where the graphs of $l_{1}$ and $l_{2}$ meet). There is $a k>0$ and a smooth function $\varepsilon$ on $[A, B]$ so that:

1) $\varepsilon^{\prime}(A)=a$,

2) $\varepsilon^{\prime}(B)=c$, and

3) $\varepsilon^{\prime \prime}(t)>k$ on $[A, B]$.

Proof. One way to do this is to start with the circle

$$
S=\left\{(x, y) \mid(x-1)^{2}+(y-1)^{2}=1\right\},
$$

and let $M: \mathbb{R}^{2} \rightarrow \mathbb{R}^{2}$ be the unique affine map taking $(0,1)$ to $\left(A, l_{1}(A)\right)$, $(1,0)$ to $\left(B, l_{2}(B)\right)$, and $(0,0)$ to the point of intersection of the graphs of $l_{1}$ and $l_{2}$. The resulting ellipse $M(S)$ is tangent to the graph of $l_{1}$ at $\left(A, l_{1}(A)\right)$, and to the graph of $l_{2}$ at $\left(B, l_{2}(B)\right)$. The part of the ellipse between these two points and closest to the union of the graphs of $l_{1}$ and $l_{2}$ is the graph of a function $\varepsilon$ satisfying the requirements of the lemma. See Figure 3 for an illustration. q.e.d.
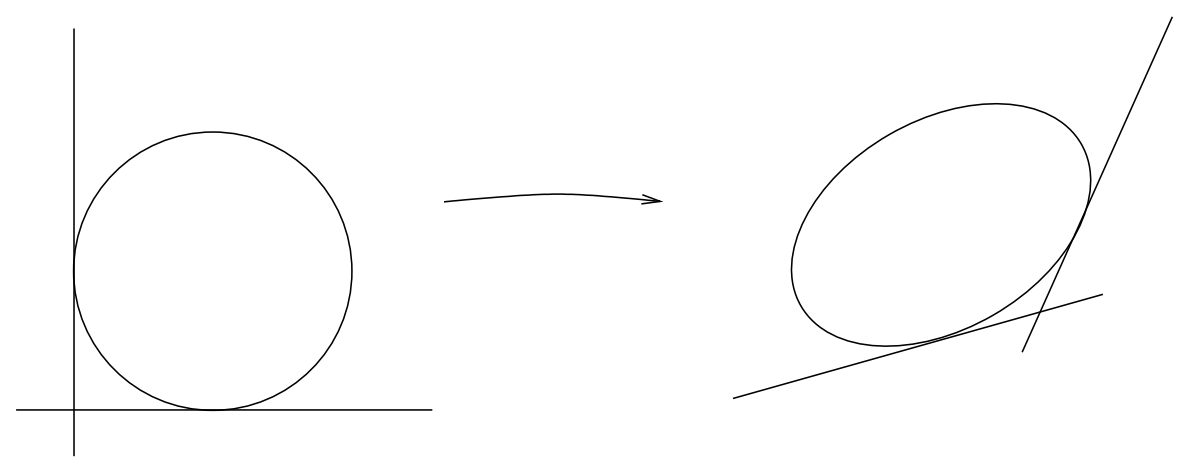

Figure 3. The curve on the right closest to the lines is a $C^{1}$ interpolation between the lines.

Proposition 4.14. For any $\lambda>0$ there is $a \delta>0, k>0$, and $a$ pair of smooth functions $f: \mathbb{R}_{+} \rightarrow \mathbb{R}_{+}$and $g: \mathbb{R}_{+} \rightarrow \mathbb{R}_{+}$satisfying: 
1) for $r<\delta, f(r)=\sinh (r)$ and $g(r)=\cosh (r)$,

2) for $r>1+\frac{\lambda}{2}, f(r)=g(r)=e^{r-1}$, and

3) for $r>0, f^{\prime \prime}(r)>0$, and for $r \in \mathbb{R}_{+}, g^{\prime \prime}(r)>k$.

Proof. We first define $C^{1}$ functions $f_{0}$ and $g_{0}$ with the above properties. We then invoke Agol's gluing lemma (Lemma 4.12) to obtain smooth functions.

Let $l_{2}$ be the equation of the tangent line to $h(r)=e^{r-1}$ at $r=1+\frac{\lambda}{2}$, and note that $l_{2}\left(\frac{\lambda}{2}\right)=0$. Since $h\left(1+\frac{\lambda}{2}\right)=e^{\frac{\lambda}{2}}>1+\frac{\lambda}{2}$, the line $l_{2}$ intersects the tangent lines at 0 to both $\cosh (r)$ and $\sinh (r)$ somewhere in the interval $\left(0,1+\frac{\lambda}{2}\right)$. By continuity, there is some $\delta_{0}>0$ so that the tangent lines to $\cosh (r)$ and $\sinh (r)$ at $r=\delta_{0}$ still hit $l_{2}$ somewhere in the interval $\left(0,1+\frac{\lambda}{2}\right)$.

In particular, if $l_{1}$ is the tangent line to $\sinh (r)$ at $r=\delta_{0}$, we may apply Lemma 4.13 to obtain a function $\varepsilon_{f}$ on $\left(\delta_{0}, 1+\frac{\lambda}{2}\right)$ with $\varepsilon_{f}^{\prime}\left(\delta_{0}\right)=$ $\cosh \left(\delta_{0}\right), \varepsilon_{f}^{\prime}\left(1+\frac{\lambda}{2}\right)=h^{\prime}\left(1+\frac{\lambda}{2}\right)$, and $\varepsilon_{f}^{\prime \prime}>k_{f}$ everywhere, for some $k_{f}>0$. We define

$$
f_{0}(r)= \begin{cases}\sinh (r) & r \leq \delta_{0} \\ \varepsilon_{f}(r) & \delta_{0}<r<1+\frac{\lambda}{2} \\ e^{r-1} & r \geq 1+\frac{\lambda}{2}\end{cases}
$$

(see Figure 4). Choosing some positive $\epsilon<\min \left\{\delta_{0}, \frac{\lambda}{2}\right\}$, we may twice

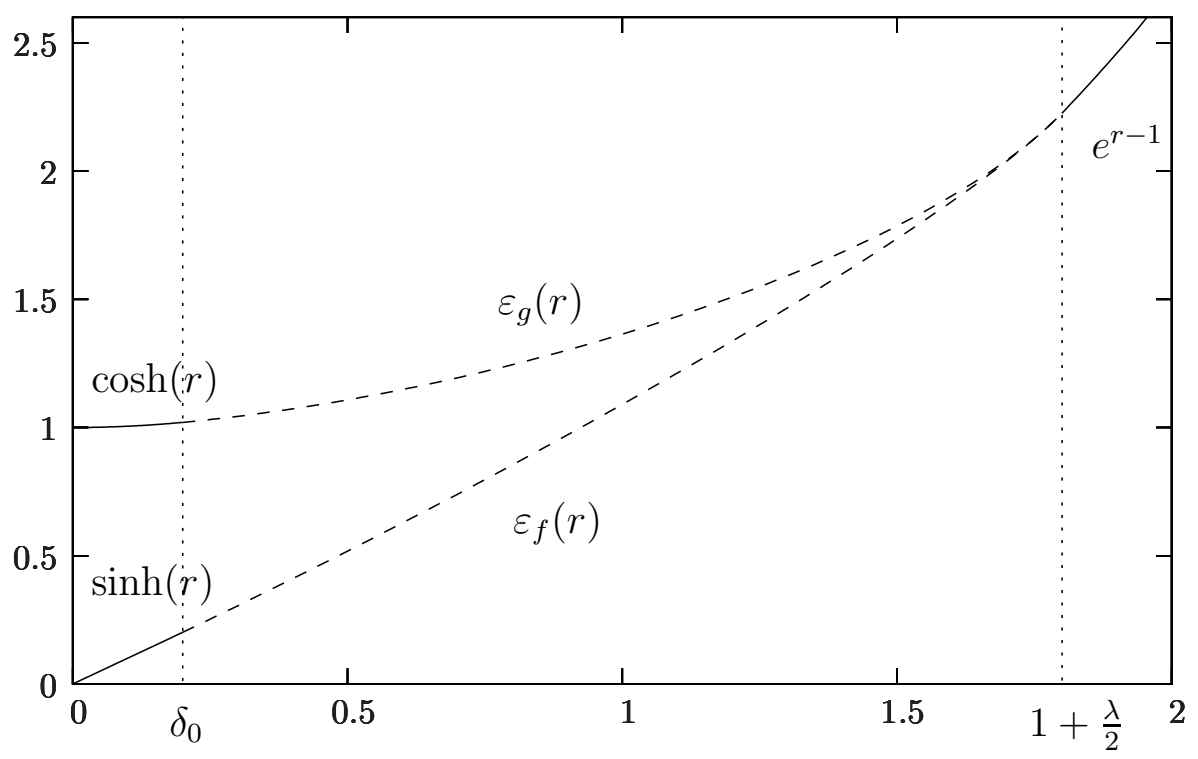

Figure 4. The bottom curve is $f_{0}$, and the top curve is $g_{0}$, for $\lambda=1.6$ and $\delta_{0}=0.2$. 
apply Lemma 4.12 (at $r=\delta_{0}$ and at $r=1+\frac{\lambda}{2}$ ) to obtain a smooth function $f: \mathbb{R}_{+} \rightarrow \mathbb{R}_{+}$satisfying:

1) $f(r)=f_{0}(r)$ outside the intervals $\left(\delta_{0}-\epsilon, \delta_{0}\right)$ and $\left(1+\frac{\lambda}{2}-\epsilon, 1+\frac{\lambda}{2}\right)$,

2) $f^{\prime \prime}(r)>0.9 \min \left\{\sinh \left(\delta_{0}\right), \varepsilon_{f}^{\prime \prime}\left(\delta_{0}\right)\right\}$ on $\left(\delta_{0}-\epsilon, \delta_{0}\right)$, and

3) $f^{\prime \prime}(r)>0.9 \min \left\{\varepsilon_{f}^{\prime \prime}\left(1+\frac{\lambda}{2}\right), e^{\frac{\lambda}{2}}\right\}$ on $\left(1+\frac{\lambda}{2}-\epsilon, 1+\frac{\lambda}{2}\right)$.

(Here 0.9 can be replaced with any number less than 1.)

A similar argument can be used to construct $g$. Lemma 4.13 can be used to construct a function $\varepsilon_{g}$ on the interval $\left(\delta_{0}, 1+\frac{\lambda}{2}\right)$ with $\varepsilon_{g}^{\prime}\left(\delta_{0}\right)=$ $\sinh \left(\delta_{0}\right), \varepsilon_{g}^{\prime}\left(1+\frac{\lambda}{2}\right)=e^{\frac{\lambda}{2}}$, and $\varepsilon_{g}^{\prime \prime}>k_{g}$ everywhere. We can thus assemble a $C^{1}$ function:

$$
g_{0}(r)= \begin{cases}\cosh (r) & r \leq \delta_{0} \\ \varepsilon_{g}(r) & \delta_{0}<r<1+\frac{\lambda}{2} \\ e^{r-1} & r \geq 1+\frac{\lambda}{2} .\end{cases}
$$

Applying Lemma 4.12 twice to $g_{0}$ and using the same $\epsilon$ as above we obtain a function $g$ satisfying:

1) $g(r)=g_{0}(r)$ outside the intervals $\left(\delta_{0}-\epsilon, \delta_{0}\right)$ and $\left(1+\frac{\lambda}{2}-\epsilon, 1+\frac{\lambda}{2}\right)$,

2) $g^{\prime \prime}(r)>0.9 \min \left\{\cosh \left(\delta_{0}\right), \varepsilon_{g}^{\prime \prime}\left(\delta_{0}\right)\right\}$ on $\left(\delta_{0}-\epsilon, \delta_{0}\right)$, and

3) $g^{\prime \prime}(r)>0.9 \min \left\{\varepsilon_{g}^{\prime \prime}\left(1+\frac{\lambda}{2}\right), e^{1+\frac{\lambda}{2}}\right\}$ on $\left(1+\frac{\lambda}{2}-\epsilon, 1+\frac{\lambda}{2}\right)$.

The functions $f$ and $g$ satisfy the conclusion of the proposition for $\delta=$ $\delta_{0}-\epsilon$, and $k=\min \left\{0.9 k_{g}, 1\right\}$.

q.e.d.

Theorem 4.15. Let $f$ and $g$ be as in Proposition 4.14, and let $T$ be flat manifold with injectivity radius bigger than $\pi$. The warped product

$$
Z=[0,1+\lambda] \times_{g(r)} \mathbb{E}^{k} \times_{f(r)} T
$$

is Riemannian away from $\{0\} \times \mathbb{E}^{k} \times T$. The space $Z$ is complete and $C A T(0)$, and is $C A T(-\kappa)$ for some $\kappa>0$ if $k \leq 1$.

Proof. Since $g(r)$ and $f(r)$ are positive and smooth for $r>0$, the space $Z$ is Riemannian away from $\Xi=\{0\} \times \mathbb{E}^{k} \times T$. Since $f(0)=0$, the space $Z$ is simply connected, so it suffices to check the curvature locally. Near the singular set $\Xi$, the $Z$ is locally isometric to a subset of the space $\tilde{F}$ described in Corollary 4.4. It follows that $Z$ is locally $\mathrm{CAT}(0)$ (and locally $\mathrm{CAT}(-1)$ if $k \leq 1$ ) in a $\delta$-neighborhood of $\Xi$.

Away from $\Xi$, we may estimate derivatives, and apply Proposition 4.11. Since $f, g$, and their first and second derivatives are all positive and continuous on the interval $[\delta, 1+\lambda]$, there is a positive lower bound $\kappa$ valid for all the quantities

$$
\frac{f^{\prime \prime}}{f}, \frac{g^{\prime \prime}}{g}, \frac{\left(f^{\prime}\right)^{2}}{f^{2}}, \frac{\left(g^{\prime}\right)^{2}}{g^{2}}, \text { and } \frac{f^{\prime} g^{\prime}}{f g}
$$

on this interval. Proposition 4.11 implies that the sectional curvatures are thus bounded above by $-\kappa$, away from a $\delta$-neighborhood of $\Xi$. 
Putting the local pictures together, $Z$ is locally (and hence globally) $\mathrm{CAT}(-\kappa)$ if $k \leq 1$, and $\mathrm{CAT}(0)$ otherwise.

q.e.d.

4.4. Nonpositively curved metrics on the partial cones. If $M$ is a hyperbolic manifold, and $E \subset M$ is a closed horospherical neighborhood of a toral cusp, then $E$ is isometric to a warped product

$$
E=(-\infty, 0] \times e^{r} N,
$$

where $N=\partial E$ with the induced flat Riemannian metric. (To see this, recall that if we identify $\mathbb{H}^{n+1}$ with $\mathbb{R} \times e_{e^{r}} \mathbb{E}^{n}$, then sets of the form $\{R\} \times \mathbb{E}^{n}$ are concentric horospheres. Any discrete group $P$ of parabolic isometries of $\mathbb{H}^{n+1}$ can be realized as a group preserving these horospheres. The quotient by the action is then of the form $\mathbb{R} \times e_{e^{r}} F$ for some flat manifold $F$. If $P$ is a maximal parabolic subgroup of a torsion-free lattice $\Gamma$, some subset of the form $(-\infty, t] \times e_{e^{r}} F$ embeds in $\mathbb{H}^{n+1} / \Gamma$ By rescaling $F$ to another flat manifold $F^{\prime}$, this subset looks like $(-\infty, 0] \times{ }_{e^{r}} F^{\prime}$.)

If $E$ is embedded in $M$, then there is some $\lambda>0$ so that $E_{\lambda}$, the closed $\lambda$-neighborhood of $E$, is still embedded. We have

$$
E_{\lambda}=(-\infty, \lambda] \times{ }_{e^{r}} N .
$$

It is convenient to reparameterize $E_{\lambda}$ as

$$
E_{\lambda}=(-\infty, 1+\lambda] \times_{e^{r-1}} N .
$$

Corresponding to any geodesic submanifold $T$ there is a cover $\tilde{N}$ of $N$, isometric to $\mathbb{E}^{k} \times T$, where $k$ is the codimension of $T$ in $N$. Likewise, there is a cover $\tilde{E}_{\lambda}$ of $E_{\lambda}$ corresponding to $T$, so that

$$
\begin{aligned}
\tilde{E}_{\lambda} & =(-\infty, 1+\lambda] \times_{e^{r-1}} \tilde{N} \\
& =(-\infty, 1+\lambda] \times_{e^{r-1}} \mathbb{E}^{k} \times_{e^{r-1}} T .
\end{aligned}
$$

The group $Q=\pi_{1}(N) / \pi_{1}(T)$ acts, preserving the product structure, by isometries on $\tilde{E}_{\lambda}$, with quotient $E_{\lambda}$. If the warping functions are replaced by any other warping functions, $\pi_{1}(N) / \pi_{1}(T)$ will still act by isometries. We will use the functions $f$ and $g$ defined in Proposition 4.14 to define a new metric, first on

$$
\tilde{W}=[0,1+\lambda] \times \mathbb{E}^{k} \times T
$$

and then on the space $W=\tilde{W} / Q$, which is homeomorphic to $C(N, T)$.

Theorem 4.16. Let $M$ be a hyperbolic $(n+1)$-manifold, with an embedded toral cusp

$$
E \cong(-\infty, 0] \times e^{r} N
$$

and suppose $T \subseteq N$ is a totally geodesic $(n-k)$-dimensional torus with injectivity radius greater than $\pi$. Choose $\lambda>0$ so that the $\lambda$ neighborhood of $E$ is still embedded in $M$, with boundary $N_{\lambda}$. 
There is then a nonpositively curved metric on the partial cone $C(N, T)$ so that the following hold:

1) $C(N, T)$ is Riemannian away from its core.

2) There is a Riemannian isometry between the $\frac{\lambda}{2}$-neighborhood of $\partial C(N, T)$, and the $\frac{\lambda}{2}-$ neighborhood of $N_{\lambda}$ in $C_{\lambda}$, which takes $T \subset$ $C(N, T)$ to a torus $T_{\lambda} \subset C_{\lambda}$, isotopic in $C_{\lambda}$ to $T$.

3) If $k \leq 1$, then $C(N, T)$ is locally $C A T(-\kappa)$ for some $\kappa>0$.

Proof. Corresponding to $T \subseteq N$ there is a cover $\tilde{N} \rightarrow N$ so that $\tilde{N}$ is isometric to $\mathbb{E}^{k} \times N$. Likewise, there is a corresponding cover of $E_{\lambda}$, which we parametrize as

$$
\tilde{E}_{\lambda}=(-\infty, 1+\lambda] \times_{e^{r-1}} \mathbb{E}^{k} \times_{e^{r-1}} T .
$$

Let $f$ and $g$ be the functions from Proposition 4.14, and let

$$
\tilde{W}=[0,1+\lambda] \times_{g(r)} \mathbb{E}^{k} \times_{f(r)} T .
$$

By Theorem $4.15, \tilde{W}$ is $\operatorname{CAT}(0)$, and is $\operatorname{CAT}(-\kappa)$ for some $\kappa>0$ if $k \leq 1$.

The group $Q=\pi_{1}(N) / \pi_{1}(T)$ acts freely and properly discontinuously by isometries on $\tilde{W}$, with quotient $W$ homeomorphic to $C(N, T)$. Thus the quotient is locally nonpositively curved, locally $\operatorname{CAT}(-\kappa)$ if $k \leq 1$, and Riemannian away from the core

$$
\left\{(0, e,-) \mid e \in \mathbb{E}^{k}\right\} / Q
$$

Note that if we write $\partial \tilde{W}$ for $\{1+\lambda\} \times \mathbb{E}^{k} \times T$, then there are $\frac{\lambda}{2}-$ neighborhoods of $\partial \tilde{W}$ and $\partial \tilde{E}_{\lambda}$ which are canonically isometric. This isometry descends to an isometry between $\frac{\lambda}{2}$-neighborhoods of $\partial W$ and $\partial E_{\lambda}$, which takes $T \subset W$ to $T_{\lambda}$ as described in the statement of the theorem.

q.e.d.

Remark 4.17. Since we obtain a compact space with the same metric near the boundary as a cusp by this operation, this is sometimes called cusp closing (cf.[40, 5, 30]). The difference in our setting is that we are not generally closing the cusp "as a manifold" or even as an orbifold. Rather, we show how to close a cusp "as a pseudomanifold", with a certain curvature control.

A reverse operation, cusp opening or "drilling", has also been studied. (See for example $[\mathbf{2}, \mathbf{2 7}]$ for applications to hyperbolic 3-manifolds.) For example, in [18] a cusp is produced under a certain curvature control after removing a totally geodesic codimension two submanifold in a closed hyperbolic manifold. This construction also used doubly warped products and was generalized in $[\mathbf{1}](\mathrm{cf} .[\mathbf{8}, \mathbf{7}])$. 
4.5. Proof of Theorem 2.7 and Proposition 2.8. Let $n \geq 2$. Suppose $M$ is a finite volume hyperbolic $(n+1)$-manifold, with horospherical cusps $E_{1}, \ldots, E_{m}$ embedded in $M$. Suppose each of those cusps is isometric to $(-\infty, 0] \times N_{i}$ for a flat torus $N_{i}$. Choose $\lambda>0$ so that the $\lambda$-neighborhood of $\bigcup_{i=1}^{n} E_{i}$ is still embedded in $M$. For each $i$, let $T_{i}$ be a totally geodesic torus in $N_{i}$ with injectivity radius larger than $\pi$, so that the space $M\left(T_{1}, \ldots, T_{m}\right)$ from Definition 2.5 is a $2 \pi$-filling of $M$.

We will describe a metric on $M\left(T_{1}, \ldots, T_{m}\right)$ satisfying the conclusions of Theorem 2.7 and 2.8 .

Fix $i \in\{1, \ldots, m\}$. The $\lambda$-neighborhood of the cusp $E_{i}$ is embedded, and disjoint from the $\lambda$-neighborhood of all the other cusps. Give $C\left(N_{i}, T_{i}\right)$ the nonpositively curved metric from Theorem 4.16.

For $t \in[0, \lambda]$, let $M_{t} \subset M$ be the closure of the complement of the union of the $t$-neighborhoods of the cusps $E_{1}, \ldots, E_{m}$. Thus $M_{0}$ is the compact manifold $\bar{M}$ from Definition 2.5. By our choice of $\lambda$, each $M_{t}$ is an embedded submanifold with boundary, and each $M_{t}$ is isotopic to $M_{0}$. Let $D_{i}$ be an open $\frac{\lambda}{2}$-neighborhood of the boundary of $C\left(N_{i}, T_{i}\right)$. According to Theorem 4.16.(2), there is an isometry

$$
\psi_{i}: D_{i} \longrightarrow M_{\frac{\lambda}{2}} \backslash M_{\lambda}
$$

for each $i$, so that the space

$$
Y=M_{\frac{\lambda}{2}} \bigcup_{\psi_{1} \sqcup \cdots \sqcup \psi_{m}}\left(C\left(N_{1}, T_{1}\right) \sqcup \cdots \sqcup C\left(N_{m}, T_{m}\right)\right)
$$

is homeomorphic to the filling $M\left(T_{1}, \ldots, T_{m}\right)$ by a homeomorphism taking $M_{\frac{\lambda}{2}} \subset Y$ isometrically to $M_{\frac{\lambda}{2}} \subset M\left(T_{1}, \ldots, T_{m}\right)$. It is clear that Proposition 2.8 holds for this metric on $M\left(T_{1}, \ldots, T_{m}\right)$, and $\bar{M}^{\prime}=M_{\frac{\lambda}{2}}$.

Conclusion (1) of Theorem 2.7 holds by construction; indeed, every non-singular point of $Y$ has a neighborhood which is either isometric to a neighborhood in $M$ or to a neighborhood in the non-singular part of some $C\left(N_{1}, T_{1}\right)$, endowed with the metric from Theorem 4.16. This metric is Riemannian by Theorem 4.16.(1). The fact that the metric on $Y$ is the completion of the Riemannian metric on the non-singular part can be deduced from Lemma 4.5.

Every point in $Y$ has a neighborhood isometric either to a neighborhood of a point in $M$, which has curvature bounded above by -1 by assumption, or in $C\left(N_{1}, T_{1}\right)$, which is nonpositively curved by Theorem 4.16. Conclusion (2) of Theorem 2.7 is established.

If for every $i$, the torus $T_{i}$ has codimension at most 1 in $N_{i}$, then the spaces $C\left(N_{i}, T_{i}\right)$ have curvature bounded above by $-\kappa$ for some $\kappa>0$, by Theorem 4.16.(3). Conclusion (3) of Theorem 2.7 follows.

4.6. Isolated flats. Let $M\left(T_{1}, \ldots, T_{m}\right)$ be a $2 \pi$-filling of the hyperbolic $(n+1)$-manifold $M$. In this section we concentrate on the case in which, for at least one $i \in\{1, \ldots, m\}$, the torus $T_{i}$ has dimension 
at most $n-2$. In this case the metric constructed in Theorem 2.7 is nonpositively curved but not locally $\operatorname{CAT}(-\kappa)$ for any positive $\kappa$. This is because the filling core $V_{i}$ of $C\left(N_{i}, T_{i}\right)$ is an isometrically embedded $k$-torus, where $k=n-\operatorname{dim}\left(T_{i}\right)>1$.

The metric described in Theorem 2.7 lifts to a metric on the universal cover $X$ of $M\left(T_{1}, \ldots, T_{m}\right)$.

Definition 4.18. A convex subset of a geodesic metric space which is isometric to $\mathbb{E}^{d}$ is called a flat, if $d \geq 2$.

The preimage of $V_{i}$ in $X$ is a union of $k$-dimensional flats. Conversely, every flat in $X$ is in the preimage of some filling core, since the metric on $M\left(T_{1}, \ldots, T_{m}\right)$ is Riemannian and negatively curved away from the cores, by Theorem 2.7.(1).

In [29], Hruska and Kleiner give a definition of isolated flats (their (IF1)), which is easier to check than the equivalent definition from [28]. The next two definitions are taken from [29].

Definition 4.19. Let $X$ be a proper metric space, and let $\mathcal{C}$ be the set of closed subsets of $X$, and let $d_{H}$ be the Hausdorff distance on $\mathcal{C}$. Let $C \in \mathcal{C}$, let $x \in X$, and let $r$ and $\epsilon$ be positive real numbers. Define

$$
U(C, x, r, \epsilon)=\left\{D \in \mathcal{C} \mid d_{H}(C \cap B(x, r), D \cap B(x, r)) \leq \epsilon\right\} .
$$

The topology of Hausdorff convergence on bounded sets is the smallest topology on $\mathcal{C}$ so that all such sets are open.

Definition 4.20. Let $X$ be a $\mathrm{CAT}(0)$ space, and let $\Gamma$ act properly discontinuously, cocompactly, and isometrically on $X$. The space $X$ has isolated flats if it contains a family of flats $\mathcal{F}$ satisfying:

- Equivariance: The set of flats $\mathcal{F}$ is invariant under the action of $\Gamma$.

- Maximality: There is a constant $B$ such that every flat in $X$ is contained in a $B$-neighborhood of some flat $F^{\prime} \in \mathcal{F}$.

- Isolation: The set $\mathcal{F}$ is a discrete subset of $\operatorname{Flat}(X)$, the set of all flats in $X$ with the topology of Hausdorff convergence on bounded sets.

We prove that our $X$ has isolated flats.

Proposition 4.21. Let $M\left(T_{1}, \ldots, T_{m}\right)$ be a $2 \pi$-filling of the hyperbolic manifold $M$, endowed with the nonpositively curved metric from Theorem 2.7. If $X$ is the universal cover of $M\left(T_{1}, \ldots, T_{m}\right)$, then $X$ has isolated flats.

Proof. We let $\mathcal{F}$ be the collection of all flats in $X$.

Maximality and equivariance are clear. We show the isolation property. Let $F_{1}$ and $F_{2}$ be elements of $\mathcal{F}$. Any path from $F_{1}$ to $F_{2}$ must project to a path from some filling core $V_{1}$ to some (possibly the same) 
filling core $V_{2}$, which passes through $\bar{M}^{\prime} \subset M\left(T_{1}, \ldots, T_{m}\right)$, where $\bar{M}^{\prime}$ is as in the statement of Proposition 2.8. Such a path must have length at least $2+\lambda$.

Let $F \in \mathcal{F}$, and let $x \in F$. Choose any $r>0$, and let $U=U(F, x, r, \lambda)$ be defined as in Definition 4.19. Clearly $F \in U$, so $U$ is an open neighborhood of $F$. On the other hand, since any path from $F$ to another flat has length at least $2+\lambda$, no other flat is in $U$, so $F$ is an isolated point of $\mathcal{F}$. The flat $F$ was arbitrary, so $\mathcal{F}$ is discrete.

q.e.d.

\section{Visual boundaries of fillings}

We suppose that $Y=M\left(T_{1}, \ldots, T_{m}\right)$ is obtained by filling a hyperbolic $(n+1)$-manifold with $m$ toral cusps, and that the tori $T_{i}$ all satisfy the hypotheses of Theorem 2.7. It follows (Corollary 2.11) that the universal cover $X$ of $M\left(T_{1}, \ldots, T_{m}\right)$ is a CAT(0) space. In particular, since $X$ is $\operatorname{CAT}(0)$, the visual boundary $\partial X$ is a boundary for $G=\pi_{1}(Y)$ in the sense of Bestvina [10]. The shape of such a boundary is an invariant of $G$ [10, Proposition 1.6]. (Since $X$ is either CAT $(-1)$ or $\operatorname{CAT}(0)$ with isolated flats, the homeomorphism type of the boundary is an invariant of $G$, by a result of Hruska [28].) In particular, the reduced integral Cech cohomology groups of $\partial X$ are invariants of $G$, since they are shape invariants. By another result of Bestvina, these cohomology groups are the same as the (reduced) cohomology groups of $G$ with $\mathbb{Z} G$ coefficients ([10, Proposition 1.5], cf. [11] for the case when $G$ is word hyperbolic). The particular results we need from $[\mathbf{1 0}]$ can be summarized:

Theorem 5.1. [10] If $G$ is the fundamental group of a compact nonpositively curved space $Y$ with universal cover $X$, and $Z$ is the visual boundary of $X$, then

$$
\check{H}^{q}(Z ; \mathbb{Z}) \cong H^{q+1}(G ; \mathbb{Z} G) .
$$

Moreover, if $G$ is a Poincaré duality group of dimension $n$, then $Z$ is a Cech cohomology sphere of dimension $n-1$.

In our setting, we do not find the homeomorphism type of $Z$, but we give enough information to determine the shape of $Z$.

5.1. The boundary as an inverse limit. If $X$ is any CAT(0) space, and $x \in X$, then the visual boundary of $X$ is homeomorphic to an inverse limit of metric spheres around $x$ [13, II.8.5]. In order to describe the visual boundary, it therefore suffices to describe

1) the spheres of finite radius about a fixed point, and

2) the projection maps toward the fixed point between these spheres.

For the remainder of this subsection, we suppose that $M$ is a hyperbolic $(n+1)$-manifold, and that $M\left(T_{1}, \ldots, T_{m}\right)$ is a filling satisfying the hypotheses of Theorem 2.7, so that $F_{1}, \ldots, F_{m}$ are the filling cores. Let $X$ be the universal cover of $M\left(T_{1}, \ldots, T_{m}\right)$. 
Definition 5.2. If $A$ is a component of the preimage in $X$ of a filling core in $M\left(T_{1}, \ldots, T_{m}\right)$, we say that $A$ is a singular point, singular geodesic, or singular flat, depending on whether $A$ is 0,1 , or $k$-dimensional for $k \geq 2$. The union of all singular points, geodesics, and flats in $X$ is called the singular set, or $\Xi$.

Definition 5.3. If $A$ is a component of the singular set, then by the construction of Section $4, A$ has a regular neighborhood in $X$ isometric to

$$
[0, \delta] \times{ }_{\cosh (t)} A \times_{\sinh (t)} T^{k}
$$

for some flat torus $T^{k}$ with injectivity radius strictly larger than $\pi$. We call this a standard neighborhood of $A$ of radius $\delta$.

Remark 5.4. In this section the emphasis is on filling cores which are positive dimensional, but all statements we give remain true, if the following conventions are kept in mind:

1) The 0 -disk is a point.

2) The -1-sphere and the -1 -disk are both the empty set $\emptyset$.

3) The spherical join of the empty set with any metric space $(M, d)$ is taken to be $\left(M, d_{\pi}\right)$, where $d_{\pi}(x, y)=\min \{\pi, d(x, y)\}$.

If all cores are assumed to be zero-dimensional, the results here merely recapitulate those of $[\mathbf{3 7}]$.

The space $X$ is a manifold away from the singular set $\Xi$. Even near the singular set, $X$ is quite well behaved.

Lemma 5.5. There exists a constant $\delta$ so that $\operatorname{loginj}(p) \geq \delta$ for all $p \in \Xi$. For $p \in X \backslash \Xi$ we have $\operatorname{loginj}(p) \geq d(p, \Xi)>0$.

Proof. If $p \in A$ for some singular component $A$ of $\Xi$, and $A$ has a standard $\delta$-neighborhood as in Definition 5.3, then $\operatorname{loginj}(p) \geq \delta$, by Corollary 4.9. Since there are finitely many orbits of components of $A$ in $X$, some $\delta$ works for all such $p$.

Let $p \in X \backslash \Xi$, let $\epsilon>0$, and let $N$ be an open $\epsilon$-neighborhood of $\Xi$ in $X$. The space $X \backslash N$ is a complete nonpositively curved manifold with boundary. If $r<d(p, \Xi)-\epsilon$, then the $r$-ball around $p$ lies in the interior of this manifold, and so the $\operatorname{logarithm} \log _{p}$ restricted to this ball is injective.

q.e.d.

Remark 5.6. We will see in Lemma 5.16 that if $p$ is not in $\Xi$, then $\operatorname{loginj}(p) \leq d(p, \Xi)$, so actually $\operatorname{loginj}(p)=d(p, \Xi)$.

Lemma 5.7. $X$ has the geodesic extension property.

Proof. Away from the singular set, $X$ is a Riemannian manifold of nonpositive curvature, so local geodesics can be extended in $X \backslash \Xi$. A local geodesic terminating on $\Xi$ can be extended by Lemma 4.7. q.e.d. 
For the remainder of the section, we fix a point $x_{0} \in X \backslash \Xi$, and a constant $\delta>0$ as in Lemma 5.5.

Definition 5.8. A sphere centered at $x_{0}$ is a metric sphere

$$
S_{r}=\left\{x \in X \mid d\left(x, x_{0}\right)=r\right\}
$$

for some $r>0$. We write $B_{r}$ for the closed ball of radius $r$ centered at $x_{0}$, and write $\stackrel{\circ}{B}_{r}$ for the interior of $B_{r}$.

For small enough $r, S_{r}$ is homeomorphic to an ordinary sphere $S^{n}$ and $B_{r}$ to an ordinary $(n+1)$-dimensional ball. Describing the topology for larger $r$ is the main goal in this subsection.

Recall that an ENR, or Euclidean Neighborhood Retract, is any space homeomorphic to a retract of an open subset of some euclidean space. Equivalently, an ENR is any locally compact, metrizable, separable, finite dimensional ANR.

Lemma 5.9. Suppose that $d\left(x_{0}, A\right) \neq r$ for any component $A$ of the singular set $\Xi$. Then the metric sphere $S_{r}$ is an ENR, and $S_{r} \backslash \Xi$ is an $n$-manifold which is open and dense in $S_{r}$.

Proof. We first note that $S_{r}$ is finite dimensional, as it is embedded in the $(n+1)$-dimensional space $X$. It is obviously locally compact, metrizable, and separable, so we need only establish that $S_{r}$ is an ANR to prove the first part of the lemma. A theorem of Dugundji [16] states that a finite dimensional metric space is an ANR if and only if it is locally contractible. We therefore examine the local behavior of $S_{r}$. Let $p \in S_{r}$.

Claim 5.10. There is some $q \in \stackrel{\circ}{B}_{r}$ so that $p$ is contained in the interior of a log-injective ball centered at $q$.

Proof. There are two cases, depending on whether $p$ is in the singular set $\Xi$ or not. Suppose first that $p \notin \Xi$, and let $d=d(p, \Xi)$. Let $q \neq p$ be a point on the geodesic from $p$ to $x_{0}$, so that $d(p, q)<d / 3$. The point $q$ is no closer than $\frac{2}{3} d$ from $\Xi$, so the log-injectivity radius at $q$ is at least $\frac{2}{3} d<d(p, q)$, by Lemma 5.5. It follows that $p$ is in a log-injective ball about $q$.

In the second case, $p$ is contained in some component $A$ of the singular set. By the hypothesis of the lemma $d\left(x_{0}, A\right)<r$; let $z \in A$ satisfy $d\left(x_{0}, z\right)<r$. Choose $q \neq p$ on the geodesic from $p$ to $z$ so that $d(p, q)<$ $\delta$, where $\delta$ is as in Lemma 5.5. Since $A$ is convex, $q \in A$. Applying Lemma 5.5, the log-injectivity radius at $q$ is at least $\delta$, and so $p$ is contained in a log-injective ball centered at $q$.

q.e.d.

Let $U$ be the log-injective ball around $q$ given by the claim, and let $B$ be a slightly smaller closed ball around $q$ so that $p$ is still in the interior of $B$. The intersection $C=B \cap B_{r}$ is a strictly convex set containing 
$q$ in its interior, so that $C$ is contained in a log-injective neighborhood of $U$. Moreover, $X$ has the geodesic extension property by Lemma 5.7. We can therefore apply Lemma 3.12 to obtain a homeomorphism

$$
h: \text { Front }(C) \rightarrow \Sigma_{q}
$$

from the frontier of $C$ to the space of directions $\Sigma_{q}$ at $q$. This space of directions is always either a round $n$-sphere or (using Lemma 4.6) an $n$-pseudomanifold isometric to the join of a sphere and a torus. In particular, it is locally contractible.

Let $s$ be the radius of the ball $B$, and choose a positive $\epsilon<s-d(p, q)$. The open $\epsilon$-ball around $p$ intersects the frontier of $C$ in a set $V \subset S_{r}$, which is an open neighborhood of $p$ in $S_{r}$. Using the homeomorphism $h$ to the locally contractible space $\Sigma_{q}$, we may find an open contractible $V^{\prime} \subset V$ containing $p$.

To see that $S_{r} \backslash \Xi$ is a manifold, note that, in the argument just made, if $p \notin \Xi$, then $q \notin \Xi$, and $h$ takes a neighborhood of $p$ in $S_{r}$ to an open set in the sphere $S^{n}$. Even for $p \notin \Xi$, the local description makes it clear that $p$ is in the closure of the set of manifold points of $S_{r}$.

q.e.d.

Definition 5.11. For any $r$, there is a map $p_{r}$ from the exterior of $S_{r}$ to $S_{r}$ defined by geodesic projection toward $x_{0}$ : If $x$ is any point lying further than $r$ from $x_{0}$, then the geodesic from $x$ to $x_{0}$ intersects $S_{r}$ in the unique point $p_{r}(x)$.

Definition 5.12. A shell is a set of the form

$$
S_{a}^{b}=\left\{x \in X \mid a \leq d\left(x, x_{0}\right) \leq b\right\}=B_{b} \backslash \stackrel{\circ}{B}_{a} .
$$

A shell $S_{a}^{b}$ is good if it satisfies all of the following:

1) If $\delta$ is the constant fixed after Lemma 5.5, then $b-a<\delta$.

2) Whenever $A$ is an $l$-dimensional component of $\Xi$ and $c \in\{a, b\}$, then $A \cap S_{c}$ is either empty or a (topological) sphere of dimension $l-1$.

3) The map $p_{a}$ restricted to $\Xi \cap S_{a}^{b}$ is an embedding.

The following observation will be useful later.

Lemma 5.13. Suppose that $a \leq s<t \leq b$. If $S_{a}^{b}$ is good, then either $S_{s}^{t}$ is good, or $d\left(x_{0}, A\right) \in\{s, t\}$ for some component $A$ of the singular set $\Xi$.

Components of the singular set have very nice intersections with good shells:

Lemma 5.14. Suppose $S_{a}^{b}$ is a good shell. Let $A$ be a component of the singular set of dimension $l$ which intersects $S_{a}^{b}$ nontrivially. If $A$ intersects $S_{a}^{b}$ but not $S_{a}$, then $A \cap S_{a}^{b}$ is a disk of dimension l. Otherwise $A \cap S_{a}$ is a sphere of dimension $l-1$ and there is a homeomorphism $h_{a, b, A}$ from $A \cap S_{a}^{b}$ to $\left(A \cap S_{a}\right) \times I$. 
Proof. Distance to the basepoint $d\left(-, x_{0}\right)$ is a proper strictly convex function on $A \cong \mathbb{E}^{l}$. If $A$ does not intersect $S_{a}$, then $A \cap S_{a}^{b}$ is a sublevel set of this strictly convex function, so $A \cap S_{a}^{b}$ is strictly convex. Since $d\left(A, x_{0}\right)<b$, the set $A \cap S_{a}^{b}$ has nonempty interior (as a subset of $A$ ), so it is an $l$-disk.

Now suppose that $A$ intersects $S_{a}$ as well as $S_{b}$. Let $q$ be the closest point on $A$ to $x_{0}$. We define the first coordinate of

$$
h_{a, b, A}: A \cap S_{a}^{b} \rightarrow\left(A \cap S_{a}\right) \times I
$$

by geodesic projection toward $q$; the second coordinate is the distance to $x_{0}$, affinely rescaled to lie in $[0,1]$. Explicitly,

$$
h_{a, b, A}(p)=\left(z, \frac{d\left(p, x_{0}\right)-a}{b-a}\right),
$$

where $z$ is the unique point in $S_{a}$ on a geodesic from $p$ to $q$. The convexity of $A$ and of the distance function make this map a homeomorphism.

q.e.d.

The next lemma says that there are plenty of good shells.

Lemma 5.15. For every $r>0$ there is some $\epsilon>0$ so that the shell $S_{r-\epsilon}^{r+\epsilon}$ is good.

Proof. Let $\delta$ be the smallest radius of a standard neighborhood of a component of the singular set of $X$. The distance between two distinct components is thus greater than $2 \delta$. It follows that if $\epsilon<\delta / 2$, then

$$
p:=\left.p_{r-\epsilon}\right|_{\Xi \cap S_{r-\epsilon}^{r+\epsilon}}
$$

cannot send points from different singular components to the same point in $S_{r-\epsilon}$.

Let $\mathcal{A}$ be the collection of components of the singular set $\Xi$, and note that the set

$$
S:=\left\{s \mid \exists A \in \mathcal{A}, d\left(x_{0}, A\right)=s\right\}
$$

is a discrete subset of $[0, \infty)$. We may therefore choose $\epsilon<\min \{\delta / 2, r\}$ so that neither $r-\epsilon$ nor $r+\epsilon$ is in $S$. Condition (1) is obviously satisfied for any such $\epsilon$. We will show that (2) and (3) are as well.

Suppose $A \in \mathcal{A}$ intersects $S_{c}$ nontrivially for $c \in\{r+\epsilon, r-\epsilon\}$, and let $B=B_{c} \cap A$ be the intersection of $A$ with the ball of radius $c$ around $x_{0}$. Since $c>d\left(A, x_{0}\right)$, the set $B$ has non-empty interior (as a subset of $A$ ), and since both $A$ and the metric are convex, $B$ is a strictly convex subset of $A$. Lemma 3.12 implies that $\partial B=A \cap S_{c}$ is an $(l-1)$-sphere, so condition (2) of Definition 5.12 is satisfied.

Finally, suppose that $S_{r-\epsilon}^{r+\epsilon}$ does not satisfy condition (3) of Definition 5.12. There is then some $A \in \mathcal{A}$ and two points $x, y \in A \cap S_{r-\epsilon}^{r+\epsilon}$ so that $p(x)=p(y)$. Since $p(x)=p(y)$, the geodesics $\left[x_{0}, x\right]$ and $\left[x_{0}, y\right]$ must coincide on some (maximal) initial segment $\left[x_{0}, z\right]$ of length at least $r-\epsilon$. 
There are two cases:

Case 1: Suppose that $z \in\{x, y\}$. Without loss of generality, suppose that $z=x$, and rechoose $x$ so that $x$ is the closest point on $\left[x_{0}, x\right] \cap A=\{x\}$. Since $A$ is convex, the geodesic $[x, y]$ lies entirely in $A$, so $[x, y]$ represents a point in $S^{n-k-1} \subset S^{n-k-1} * T^{k}=\Sigma_{x}$. Since $\left[x_{0}, y\right]$ is geodesic, the geodesic sub-segments $[x, y]$ and $\left[x, x_{0}\right]$ must have Alexandroff angle $\pi$ at $x$. This implies that $\left[x, x_{0}\right]$ also lies in $S^{n-k-1} \subset S^{n-k-1} * T^{k}=\Sigma_{x}$, but since $A$ has a standard neighborhood, this implies that $\left[x, x_{0}\right]$, and hence $x_{0}$, lies in $A$. But this contradicts the fact that $x_{0}$ lies outside the singular set.

Case 2: Suppose $z \notin\{x, y\}$. The directions in $\Sigma_{z}$ corresponding to $[z, x]$ and $[z, y]$ are both at distance $\pi$ from the direction corresponding to $\left[z, x_{0}\right]$. Since the logarithm map is injective near $z$, these directions are all distinct in $\Sigma_{z}$. In particular, $\Sigma_{z}$ is not a round sphere of diameter $\pi$ and so $z$ must lie in some component of the singular set $A^{\prime}$. Since $p(z)=p(x)=p(y)$, we must have $A^{\prime}=A$. Replacing either $x$ or $y$ by $z$, we may derive a contradiction as in Case 1.

This establishes condition (3).

q.e.d.

To understand the difference between the two boundary components of a good shell, we have to understand how geodesics can be continued through a piece of the singular set. In a simply connected Riemannian manifold of non-positive curvature, geodesics (in the sense of lengthminimizing paths) can always be continued uniquely. At a point in a singular space whose space of directions is not a round sphere of diameter $\pi$ (or a point which does not have a log-injective neighborhood) this uniqueness generally fails. The next lemma gives a description of the collection of geodesics of a fixed length starting at a point and passing through some particular component of the singular set.

Lemma 5.16. Let $A$ be a component of the singular set isometric to $\mathbb{E}^{l}$, and let $k=n-l$. Choose $R$ so that

$$
d\left(x_{0}, A\right)<R<d\left(x_{0}, A\right)+\delta .
$$

If $Z$ is the set of endpoints of geodesics of length $R$ starting at $x_{0}$ and meeting $A$, then $Z$ is homeomorphic to $S^{l-1} * P^{k}$, where $P^{k}$ is a flat $k$-dimensional torus minus the interior of a closed embedded $k$ dimensional ball.

Proof. By the construction of Section 4, there is a standard neighborhood of $A$ which we may identify with the warped product:

$$
[0, \delta] \times{ }_{\cosh (r)} A \times \times_{\sinh (r)} T
$$

for some flat $k$-torus $T$ whose injectivity radius is greater than $\pi$. Let $a \in A$ be the closest point to $x_{0}$, and let $a^{\prime} \in A$ be arbitrary. Via 
Proposition 3.7, we may identify the space of directions $\Sigma_{a^{\prime}}(X)$ with $\Sigma_{a}(A) * T$. Let

$$
\pi_{T}: \Sigma_{a^{\prime}}(X) \backslash \Sigma_{a^{\prime}}(A) \rightarrow T
$$

be the canonical projection onto the $T$ factor. Finally, let $\psi: Z \rightarrow A$ send a point $z \in Z$ to the unique point in $A$ on the geodesic from $z$ to $x_{0}$. by

We can then define a map $\phi: Z \rightarrow \Sigma_{a}(A) * T=\left[0, \frac{\pi}{2}\right] \times \Sigma_{a}(A) \times T / \sim$

$$
\phi(z)=\left(\frac{\pi}{2} \frac{d(\psi(z), z)}{R-d\left(x_{0}, a\right)},[a, \psi(z)], \pi_{T}([\psi(z), z])\right),
$$

abusing notation in the second and third factor by writing a geodesic segment with a given direction instead of the direction itself. Because geodesics are continuous as their endpoints are varied, $\phi$ is a continuous map onto some closed subset of $\Sigma_{a}(A) * T$. Note here that the first two factors are essentially the polar coordinates centered at $a$ of $\psi(z)$, reparametrized in the radial direction. To check injectivity of $\phi$, we therefore only need to check injectivity of $\phi$ restricted to $\psi^{-1}\left(a^{\prime}\right)$ for $a^{\prime} \in A \cap B_{R}$. If $d\left(a^{\prime}, x_{0}\right)=R$, there is nothing to check, so it suffices to verify the following.

Claim 5.17. For $a^{\prime} \in A$ with $d\left(a^{\prime}, x_{0}\right)<R$, $\phi$ restricted to $\psi^{-1}\left(a^{\prime}\right)$ is an embedding, and $\psi^{-1}\left(a^{\prime}\right)$ is homeomorphic to $T$ minus an open ball of radius $\pi$.

Proof. For any $z \in \psi^{-1}\left(a^{\prime}\right)$, the geodesic from $x_{0}$ to $z$ is composed of two subsegments $\left[a^{\prime}, x_{0}\right]$ and $\left[a^{\prime}, z\right]$. Regarded as directions in $\Sigma_{a^{\prime}}(X)$, these subsegments must subtend an angle of $\pi$. Conversely, any direction which is Alexandroff angle $\pi$ from $\left[a^{\prime}, x_{0}\right]$ is the direction of the geodesic continuation of $\left[x_{0}, a^{\prime}\right]$ on to some unique $z \in Z$. (Uniqueness follows from log-injectivity at $a^{\prime}$ of the $\delta$-neighborhood of $A$, in which $Z$ is contained.) To sum up, $\log _{a^{\prime}}$ is an embedding restricted to $\psi^{-1}\left(a^{\prime}\right)$, with image $D$, the set of directions whose angle with $\left[a^{\prime}, x_{0}\right]$ is exactly $\pi$.

Identifying $\Sigma_{a^{\prime}}(X)$ with $\Sigma_{a}(A) * T$, suppose that $\left[a^{\prime}, x_{0}\right]=(\phi, \alpha, \theta)$ in the standard join coordinates. Because $x_{0} \notin A, \phi \neq 0$. Standard trigonometric identities can be used to show that those $\left(\phi^{\prime}, \alpha^{\prime}, \theta^{\prime}\right)$ which make an angle of $\pi$ with $\left[a^{\prime}, x_{0}\right]$ are exactly those with $\phi^{\prime}=\phi, \alpha^{\prime}=-\alpha$, and $d\left(\theta^{\prime}, \theta\right) \geq \pi$. It follows that $\pi_{T}\left(\log _{a^{\prime}}\left(\psi^{-1}\left(a^{\prime}\right)\right)\right)$ is $T$ minus an open ball of radius $\pi$ around $\theta$.

q.e.d.

It follows that the image of $\phi$ is homeomorphic to the join of $\Sigma_{a}(A)$ with $T$ minus a ball of radius $\pi$. Since $\phi$ was a homeomorphism onto its image, and $\Sigma_{a}(A)$ is an $(l-1)$-sphere, the lemma is proved. q.e.d.

Remark 5.18. The space $S^{l-1} * P^{k}$ described in Lemma 5.16 is a pseudomanifold with boundary homeomorphic to $S^{l-1} * S^{k-1} \cong S^{n-1}$. 
Definition 5.19. Let $Y_{1}$ and $Y_{2}$ be spaces containing $U_{1} \subseteq Y_{1}$ and $U_{2} \subseteq Y_{2}$ which are open, connected, dense, and homeomorphic to oriented $n$-manifolds. Then the connect sum $Y_{1} \# Y_{2}$ is the space obtained by choosing small closed $n$-balls $D_{1} \subset U_{1}$ and $D_{2} \subset U_{2}$, choosing an orientation reversing homeomorphism $\phi: \partial D_{1} \rightarrow \partial D_{2}$, and gluing the exteriors together thus:

$$
Y_{1} \# Y_{2}:=\left(Y_{1} \backslash \stackrel{\circ}{D}_{1}\right) \cup_{\phi}\left(Y_{1} \backslash \stackrel{\circ}{D}_{2}\right) .
$$

For $\{i, j\}=\{1,2\}$ there is a (unique up to homotopy) map $q_{Y_{i}}: Y_{1} \#$ $Y_{2} \rightarrow Y_{i}$ which takes $Y_{i} \backslash \stackrel{\circ}{D}_{i}$ to itself by the identity map, and sends $Y_{j} \backslash \stackrel{\circ}{D}_{j}$ onto $D_{i}$ by a degree one map. We refer to $q_{Y_{i}}$ as the map which pinches $Y_{j}$ to a point.

We remark that the operation of connect sum described in Definition 5.19 is associative, so that the connect sum of three or more spaces is well-defined. Moreover, if $W=(A \# B) \# C \cong A \#(B \# C)$, then the map which pinches $B \# C$ to a point is homotopic to the composition of the map which pinches $B$ to a point with the map which pinches $C$ to a point.

Proposition 5.20. Suppose $S_{a}^{b}$ is a good shell, and let $A_{1}, \ldots, A_{p}$ be the components of the singular set which intersect $S_{a}^{b}$ but not $S_{a}$. If the dimension of $A_{i}$ is $l_{i}=n-k_{i}$ for each $i$, then there is a homotopy equivalence $\phi$ from $S_{b}$ to the connect sum $S_{a} \# J$, where

$$
J=\stackrel{p}{\#} S_{i=1}^{l_{i}-1} * T^{k_{i}}
$$

Moreover, the projection map $p_{a}^{b}=\left.p_{a}\right|_{S_{b}}$ fits into a homotopy commutative triangle

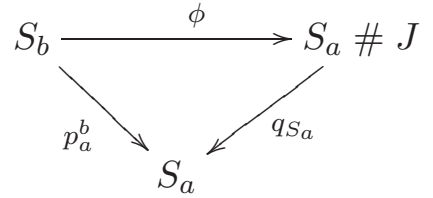

where $q_{S_{a}}$ is the map which pinches $J$ to a point.

Proof. One complicating point here is that near the components of the singular set, the projection map $p_{a}^{b}$ is not a local homeomorphism. This is true even if $S_{a}$ and $S_{b}$ intersect the same components of the singular set. To deal with this issue, we replace $S_{a}$ and $S_{b}$ with homotopy equivalent quotients $Q_{a}$ and $Q_{b}$ so that $Q_{b} \cong Q_{a} \# J$, chosen so that $p_{a}^{b}$ induces a map $\bar{p}_{Q}: Q_{b} \rightarrow Q_{a}$, and so that $p_{Q}$ is the map which pinches $J$ to a point.

The maps $h_{a, b, A}$ from Lemma 5.14 can be patched together to give an embedding $h:\left(S_{a} \cap \Xi\right) \times I \rightarrow S_{a}^{b}$ whose image is the union of the components of $\Xi \cap S_{a}^{b}$ which intersect both boundary components of the 
shell. Since $S_{a}^{b}$ is a good shell, the map $p_{a}$ restricts to an embedding of $\Xi \cap S_{a}^{b}$, and so $p_{a} \circ h$ is also an embedding.

We next give decompositions $\mathcal{G}_{a}$ and $\mathcal{G}_{b}$ of $S_{a}$ and $S_{b}$ into closed sets. A set in $\mathcal{G}_{a}$ is either a point outside the image of $p_{a} \circ h$, or it is an arc of the form $p_{a} \circ h(e \times I)$ for some $e \in S_{a} \cap \Xi$.

A set in $\mathcal{G}_{b}$ is either a point $z$ so that $p_{a}(z)$ lies outside the image of $p_{a} \circ h$, or it is the preimage of one of the arcs of the form $p_{a} \circ h(e \times$ $I)$. Arguing as in Lemma 5.16, each element of $\mathcal{G}_{b}$ is either a point or homeomorphic to the cone on a closed subset of a torus.

We leave it to the reader to check that the decompositions $\mathcal{G}_{a}$ and $\mathcal{G}_{b}$ are upper semicontinuous. It follows that $Q_{a}$ and $Q_{b}$ are Hausdorff, and therefore compact metric spaces.

The projection map from $S_{a}$ sends elements of $\mathcal{G}_{b}$ to elements of $\mathcal{G}_{a}$, and thus induces a continuous map $p_{Q}$ from $Q_{b}$ to $Q_{a}$. We have the following commuting square:

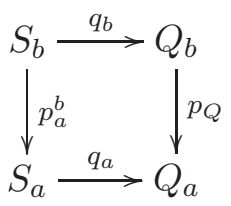

Note that the map $p_{Q}$ is a homeomorphism away from the image in $Q_{a}$ of

$$
\left(p_{a}^{b}\right)^{-1}\left(p_{a}\left(\cup_{i} A_{i} \cap S_{a}^{b}\right)\right) .
$$

The Proposition now reduces to two claims:

Claim 5.21. The space $Q_{b}$ is homeomorphic to the connect sum $Q_{a} \#$ $J$, and $p_{Q}$ is homotopic to the map which pinches $J$ to a point.

Claim 5.22. The horizontal maps in (2) are homotopy equivalences.

Proof of Claim 5.21. As noted above, $p_{Q}$ is a homeomorphism away from

$$
q_{a}\left(\left(p_{a}^{b}\right)^{-1}\left(\cup_{i=1}^{p} A_{i} \cap S_{a}^{b}\right)\right),
$$

where the $A_{i}$ are those components of the singular set which intersect $S_{a}^{b}$ but not $S_{a}$. A small regular neighborhood in $S_{a}$ of each $l_{i}$-dimensional disk $D_{i}:=p_{a}\left(A_{i} \cap S_{a}^{b}\right)$ is a ball $B_{i}$ (again using Lemma 5.14), so this implies that $Q_{b}$ is homeomorphic to the connect sum of $Q_{a}$ with some collection of spaces $\left\{K_{i} \mid 1 \leq i \leq p\right\}$ where each $K_{i}$ is equal to $\left(p_{a}^{b}\right)^{-1}\left(B_{i}\right)$, capped off with a ball.

We must describe the topology of the spaces $\left(p_{a}^{b}\right)^{-1}\left(B_{i}\right)$. The topology of $\left(p_{a}^{b}\right)^{-1}\left(D_{i}\right)$ is given in Lemma 5.16: Each such space is homeomorphic to a join $S^{l_{i}-1} * P^{k_{i}}$ with $k_{i}+l_{i}=n$ and $P$ homeomorphic to a $k_{i}$-torus with a small open ball removed. It follows that $K_{i}$ is homeomorphic to the join of a $k_{i}$-torus with an $\left(l_{i}-1\right)$-sphere. 
Putting the pieces together, we see that $Q_{b}$ is homeomorphic to a connect sum of $Q_{a}$ with the space $J$ described in the statement, and projection from $Q_{b}$ to $Q_{a}$ is homotopic to the map which pinches $J$ to a point.

q.e.d.

Proof of Claim 5.22. We first claim that the quotient spaces $Q_{a}$ and $Q_{b}$ are ENRs. Indeed, Lemma 5.9 implies that the metric spheres $S_{a}$ and $S_{b}$ are ENRs, so each of $Q_{a}$ and $Q_{b}$ is a quotient of an ENR by a decomposition into closed contractible (in particular cell-like) sets. A theorem of Lacher [34, Corollary 3.3] says that if such a quotient is metrizable and finite dimensional, then it is an ENR. We have already shown that $Q_{a}$ and $Q_{b}$ are metrizable. Moreover, there is an obvious decomposition of each into an open $n$-manifold and a union of closed annuli and disks of strictly lower dimension. (Only annuli are needed for $Q_{a}$.) Applying the Addition Theorem from dimension theory (see, e.g. $[\mathbf{1 7}, 3.1 .17])$, we obtain the finite dimensionality of $Q_{a}$ and $Q_{b}$.

It now follows from another theorem of Lacher [34, Theorem 1.2] that the quotient maps are homotopy equivalences.

q.e.d.

The Proposition now follows from the two claims.

q.e.d.

Theorem 5.23. Let $n \geq 2$. Let $M$ be a hyperbolic $(n+1)$-manifold with $m$ toral cusps $E_{1}, \ldots, E_{m}$, let $\bar{M}$ be a manifold with boundary obtained from $M$ by removing open horospherical neighborhoods of the cusps, and let $\left\{T_{1}, \ldots, T_{m}\right\}$ be totally geodesic tori in $\partial \bar{M}$ satisfying the hypotheses of Theorem 2.7. Let $s$ be the maximum of the dimensions of $\left\{T_{1}, \ldots, T_{m}\right\}$. Let $X=M\left(T_{1}, \ldots, T_{m}\right)$ be obtained from $M$ by filling along $\left\{T_{1}, \ldots, T_{m}\right\}$, and let $G=\pi_{1}(X)$.

The cohomology of $G$ with $\mathbb{Z} G$ coefficients satisfies:

$$
H^{q}(G ; \mathbb{Z} G)= \begin{cases}0 & q \leq n-s+1 \text { or } q>n+1 \\ \mathbb{Z}^{\infty} & n-s+2 \leq q \leq n \\ \mathbb{Z} & q=n+1 .\end{cases}
$$

Proof. Let $Y$ be the universal cover of $X$. By Theorem 2.7, the space $Y$ is $\operatorname{CAT}(0)$. As pointed out in [10], the visual boundary $\partial Y$ is a boundary for $G$, and so we have $H^{q}(G ; \mathbb{Z} G)=\check{H}^{q-1}(\partial Y ; \mathbb{Z})$ for all $q$.

We thus compute the Cech cohomology of $\partial Y$.

As at the beginning of Section 5.1, choose some $x_{0}$ in $Y$, not in the singular set, and write $S_{r}$ for the metric sphere of radius $r$ centered as $x$. As already noted, the visual boundary of $Y$ is homeomorphic to the inverse limit of the system of spheres centered at $x$, i.e.,

$$
\partial Y \cong \lim _{\longleftarrow}\left\{S_{t} \stackrel{p_{s}^{t}}{\longrightarrow} S_{s} \mid s, t \in \mathbb{R} \text { and } t \geq s\right\}
$$

where $p_{t}^{s}$ is projection along geodesics toward $x_{0}$. In fact, any subcollection of spheres $\left\{S_{r_{i}} \mid i \in \mathbb{N}\right\}$ with $\lim _{i \rightarrow \infty} r_{i}=\infty$ determines the 
inverse limit. By Lemmas 5.13 and 5.15, we may therefore choose the sequence $\left\{r_{i}\right\}$ so that each shell $S_{r_{i}}^{r_{i+1}}$ is good. Let $p_{i}=p_{r_{i}}^{r_{i+1}}$. We have

$$
\partial Y \cong \lim _{\longleftarrow}\left\{S_{r_{i+1}} \stackrel{p_{i}}{\longrightarrow} S_{r_{i}} \mid i \in \mathbb{N}\right\},
$$

and so

$$
\check{H}^{q}(\partial Y ; \mathbb{Z})=\lim _{\longrightarrow}\left\{H^{q}\left(S_{r_{i}} ; \mathbb{Z}\right) \stackrel{p_{i}^{*}}{\longrightarrow} H^{q}\left(S_{r_{i+1}} ; \mathbb{Z}\right) \mid i \in \mathbb{N}\right\} .
$$

For each $i$, we apply Proposition 5.20 to compute $p_{i}^{*}$. Let $J_{i}$ be the space $J$ from the proposition, applied with $b=r_{i+1}$ and $a=r_{i}$, and let $s_{i}$ be the largest dimension of any torus $T_{j}$ occurring in $J_{i}=\#_{j=1}^{p_{i}} S_{j}$ * $T_{j}$. Elementary algebraic topology shows that $p_{i}^{*}$ is injective, and that $\operatorname{coker}\left(p_{i}^{*}\right)$ is free abelian of nonzero rank in dimension $j$ for

$$
n-s_{i}+1 \leq j \leq n-1,
$$

and zero in all other dimensions. Note that $s_{i}=s$ for infinitely many $i \in \mathbb{N}$.

It follows that the (reduced) Cech cohomology of $\partial G$ is

$$
\check{H}^{q}(\partial G ; \mathbb{Z})=\left\{\begin{array}{lll}
0 & \text { if } & q \leq n-s \text { or } q>n \\
\mathbb{Z}^{\infty} & \text { if } & n-s+1 \leq q \leq n-1 \\
\mathbb{Z} & \text { if } & q=n .
\end{array}\right.
$$

Applying Theorem 5.1 gives the desired result.

q.e.d.

Corollary 5.24. Let $M,\left\{T_{1}, \ldots, T_{m}\right\}$ satisfy the hypotheses of Theorem 5.23. The group $G=\pi_{1}\left(M\left(T_{1}, \ldots, T_{m}\right)\right)$ is a Poincaré duality group if and only if every $T_{i}$ has dimension one.

Proof. If every $T_{i}$ has dimension one, then $M\left(T_{1}, \ldots, T_{m}\right)$ is a nonpositively curved (and hence aspherical) closed manifold of dimension $n+1$, and so $\pi_{1}\left(M\left(T_{1}, \ldots, T_{m}\right)\right)$ must be a Poincaré duality group of dimension $n+1$. (The special case in which every $T_{i}$ has dimension one was already handled by Schroeder in [40].)

If some $T_{i}$ has dimension different from one, then Theorem $5.23 \mathrm{im}$ plies that $G$ has cohomological dimension $n+1$, but $H^{q}(G ; \mathbb{Z} G) \neq 0$ for some $q \neq n+1$, and so $G$ cannot be an $(n+1)$-dimensional Poincaré duality group, by the second part of Theorem 5.1.

q.e.d.

Remark 5.25. The proof that our examples are not manifold groups is essentially the same as that used in [37] for the case in which every cusp is coned off completely. The difference in our setting is that we get a wider variety of behavior at infinity.

\subsection{Simple connectivity at infinity.}

Proposition 5.26. Let $M\left(T_{1}, \ldots, T_{m}\right)$ be a $2 \pi$-filling of an $(n+1)$ dimensional hyperbolic manifold $M$. Unless some $T_{i}$ is $n$-dimensional, the universal cover $X$ of $M\left(T_{1}, \ldots, T_{m}\right)$ is simply connected at infinity. 
Proof. We retain the notation of Subsection 5.1. To show simple connectivity at infinity, it suffices to show that for every $r>0$ there is some $R>r$ so that any loop in $X \backslash B_{R}$ is null-homotopic in $X \backslash B_{r}$.

Accordingly, let $r>0$, and choose $r_{1}<r_{2}<r_{3}<\cdots$ as in the proof of Theorem 5.23, so that each shell $S_{r_{i}}^{r_{i+1}}$ is good and so that $\lim _{i \rightarrow \infty} r_{i}=\infty$. Choose some $i$ so that $r_{i}>r$. We claim that $R=r_{i}$ suffices.

Let $\gamma: S^{1} \rightarrow X \backslash B_{R}$ be a loop. It is easy to show that $X$ deformation retracts (holding $B_{R}$ fixed) to $B_{R}$, so $\gamma$ is homotopic in the complement of $B_{r}$ to a loop $\gamma^{\prime}$ in $S_{R}$. Induction together with Proposition 5.20 shows that $S_{R}$ is a connect sum of joins of tori and spheres. Moreover, unless some $T_{i}$ is $n$-dimensional, each such join is simply connected, so $S_{R}$ is simply connected. It follows that $\gamma^{\prime}$ is null-homotopic in $S_{R}$, so $\gamma$ is null-homotopic in the complement of $B_{r}$.

q.e.d.

In $[\mathbf{3 8}]$, it is shown that systolic groups are never simply connected at infinity.

Corollary 5.27. Let $G=\pi_{1}\left(M\left(T_{1}, \ldots, T_{m}\right)\right)$, where $M\left(T_{1}, \ldots, T_{m}\right)$ is a $2 \pi$-filling of an $(n+1)$-dimensional hyperbolic manifold, and suppose that no $T_{i}$ is $n$-dimensional. Then $G$ is not systolic.

\section{Further questions}

Suppose we are given a group $G$ and a collection of subgroups $\mathcal{P}=$ $\left\{P_{1}, \ldots, P_{m}\right\}$ with respect to which $G$ is relatively hyperbolic. Suppose further that normal subgroups $N_{i} \triangleleft P_{i}$ (filling kernels) are given, and $K=\left\langle\left\langle\cup_{i} N_{i}\right\rangle\right\rangle_{G}$ is the normal closure in $G$ of these subgroups. The group $G\left(N_{1}, \ldots, N_{m}\right):=G / K$ is said to be a hyperbolic filling of $G$ if

1) the obvious map $\psi_{i}: P_{i} / N_{i} \rightarrow G\left(N_{1}, \ldots, N_{m}\right)$ is injective for each $i$, and

2) $G\left(N_{i}, \ldots, N_{m}\right)$ is hyperbolic relative to $\left\{\psi_{i}\left(P_{i} / N_{i}\right) \mid 1 \leq i \leq m\right\}$.

The main results of $[\mathbf{2 5}, \mathbf{3 9}]$ show that as long as every filling kernel $N_{i}$ is composed entirely of "long" elements of $P_{i}$, the group $G\left(N_{1}, \ldots, N_{m}\right)$ is always a hyperbolic filling of $G$.

If $G$ acts properly and cocompactly on a $\mathrm{CAT}(0)$ space with isolated flats, then it is hyperbolic relative to the flat stabilizers $[\mathbf{2 9}]$. These flat stabilizers are virtually abelian, and so there are many candidates for the filling kernels as above.

Question 6.1. If $G$ acts properly and cocompactly on a $C A T(0)$ space with isolated flats, does every hyperbolic filling of $G$ act properly and cocompactly on some CAT(0) space with isolated flats? What can be said about the dimension of this space?

Question 6.2. If $G$ acts properly and cocompactly on a $C A T(0)$ space with isolated flats (or geometrically finitely on a CAT(-1) space), and 
$G^{\prime}$ is a hyperbolic filling of $G$ which is hyperbolic, does $G$ act properly and cocompactly on some CAT(-1) space? What can be said about the dimension of this space?

In the current paper we have given answers to both of these questions in the special case that $G$ is the fundamental group of a hyperbolic $(n+1)$-manifold with toral cusps and the filling kernels are direct summands of the (free abelian) peripheral subgroups. It should be possible to appeal to Haefliger's theory of nonpositively curved orbispaces and extend our results to the case in which the filling kernels are arbitrary subgroups of the peripheral subgroups.

It seems much more challenging to extend the current techniques to hyperbolic fillings of fundamental groups of finite volume complex or quaternionic hyperbolic manifolds.

Focusing on fillings of the fundamental group $G$ of a fixed hyperbolic manifold $M$ with toral cusps, other kinds of question arise. Theorem 2.13 says that there are infinitely many isomorphism types of hyperbolic fillings of $M$. On the other hand, the discussion in Section 5 shows that these fillings have boundary whose shape is determined by the dimensions of the filling tori.

Question 6.3. Let $n \geq 2$. Let $M$ be a hyperbolic $(n+1)$-manifold with toral cusps, and let $A \subseteq\{1, \ldots, n\}$. Let $\mathcal{M}_{A}$ be the collection of fundamental groups of $2 \pi$-fillings $M\left(T_{1}, \ldots, T_{m}\right)$ so that

$$
\left\{\operatorname{dim}\left(T_{i}\right) \mid i \in\{1, \ldots, m\}\right\}=A .
$$

How many quasi-isometry types are there in $\mathcal{M}_{A}$ ?

The case of classical hyperbolic Dehn filling of 3-manifolds is $A=\{1\}$ and $n=2$. The $2 \pi$ theorem together with the positive solution to the geometrization conjecture by Perelman $[\mathbf{3 5}, \mathbf{3 6}]$ implies that, in this case, every group in $\mathcal{M}_{A}$ is quasi-isometric to $\mathbb{H}^{3}$.

In Mosher and Sageev's setting in [37], $A=\{n\}$ and the set $\mathcal{M}_{A}$ has at most one element.

In all other cases, Question 6.3 seems to be open.

\section{References}

[1] U. Abresch \& V. Schroeder. Graph manifolds, ends of negatively curved spaces and the hyperbolic 120-cell space. J. Differential Geom. 35(2) (1992) 299-336. MR 1158338, Zbl 0777.53035.

[2] I. Agol. Volume change under drilling. Geom. Topol. 6 (2002) 905-916. MR 1943385, Zbl 1031.57014.

[3] S. Alexander \& R. L. Bishop. $\mathcal{F} K$-convex functions on metric spaces. Manuscripta Math. 110(1) (2003) 115-133. MR 1951803, Zbl 1020.53049.

[4] S. B. Alexander \& R. L. Bishop. Curvature bounds for warped products of metric spaces. Geom. Funct. Anal. 14(6) (2004) 1143-118. MR 2135163, Zbl 1087.53064. 
[5] M. T. Anderson. Dehn filling and Einstein metrics in higher dimensions. J. Differential Geom. 73(2) (2006) 219-261. MR 2225518.

[6] I. Belegradek. Aspherical manifolds with relatively hyperbolic fundamental groups. Geom. Dedicata 129 (2007) 119-144. MR 2353987, Zbl 1139.20036.

[7] I. Belegradek. Complex hyperbolic hyperplane complements, 2007, preprint, arXiv:0711.5001.

[8] I. Belegradek. Rigidity and relative hyperbolicity of real hyperbolic hyperplane complements, 2007, preprint, arXiv:0711.2324.

[9] M. Bestvina. Questions in geometric group theory. available at http://www. math.utah.edu/ bestvina.

[10] M. Bestvina. Local homology properties of boundaries of groups. Michigan Math. J. 43(1) (1996) 123-139. MR 1381603, Zbl 0872.57005.

[11] M. Bestvina \& G. Mess. The boundary of negatively curved groups. J. Amer. Math. Soc. 4(3) (1991) 469-481. MR 1096169, Zbl 0767.20014.

[12] S. A. Bleiler \& C. D. Hodgson. Spherical space forms and Dehn filling. Topology 35(3) (1996) 809-833. MR 1396779, Zbl 0863.57009.

[13] M. R. Bridson \& A. Haefliger. Metric spaces of non-positive curvature, volume 319 of: Grundlehren der Mathematischen Wissenschaften [Fundamental Principles of Mathematical Sciences]. Springer-Verlag, Berlin, 1999. MR 1744486, Zbl 0988.53001.

[14] D. Burago, Y. Burago, \& S. Ivanov. A course in metric geometry, volume 33 of: Graduate Studies in Mathematics. American Mathematical Society, Providence, RI, 2001. MR 1835418, Zbl 0981.51016.

[15] R. M. Charney \& M. W. Davis. Strict hyperbolization. Topology 34(2) (1995) 329-350. MR 1318879, Zbl 0826.53040.

[16] J. Dugundji. Absolute neighborhood retracts and local connectedness in arbitrary metric spaces. Compositio Math. 13 (1958) 229-246. MR 0113217, Zbl 0089.38903.

[17] R. Engelking. Theory of dimensions finite and infinite, volume 10 of: Sigma Series in Pure Mathematics. Heldermann Verlag, Lemgo, 1995. MR 1363947, Zbl 0872.54002.

[18] K. Fujiwara. A construction of negatively curved manifolds. Proc. Japan Acad. Ser. A Math. Sci., 64(9) (1988) 352-355. MR 979243, Zbl 0668.53028.

[19] K. Fujiwara \& J. F. Manning. Simplicial volume and fillings of hyperbolic manifolds. in preparation.

[20] M. Gromov. Manifolds of negative curvature. J. Differential Geom. 13(2) (1978) 223-230. MR 540941, Zbl 0433.53028.

[21] M. Gromov. Volume and bounded cohomology. Inst. Hautes Études Sci. Publ. Math., 56 (1983) 5-99. MR 0686042, Zbl 0516.53046.

[22] M. Gromov. Hyperbolic groups. In: Essays in group theory, volume 8 of: Math. Sci. Res. Inst. Publ., pages 75-263. Springer, New York, 1987. MR 919829, Zbl 0634.20015.

[23] M. Gromov. Asymptotic invariants of infinite groups. In: Geometric group theory, Vol. 2 (Sussex, 1991), volume 182 of: London Math. Soc. Lecture Note Ser., pages 1-295. Cambridge Univ. Press, Cambridge, 1993. MR 1253544, Zbl 0841.20039. 
[24] D. Groves. Limit groups for relatively hyperbolic groups. II. Makanin-Razborov diagrams. Geom. Topol. 9 (2005) 2319-2358. MR 2209374, Zbl 1100.20032.

[25] D. Groves \& J. F. Manning. Dehn filling in relatively hyperbolic groups. Israel J. Math. 168 (2008) 317-429. MR 2448064, Zbl pre05508729.

[26] F. Haglund. Complexes simpliciaux hyperboliques de grande dimension. Prépublication d'Orsay, 2003.

[27] C. D. Hodgson \& S. P. Kerckhoff. Universal bounds for hyperbolic Dehn surgery. Ann. of Math. (2) 162(1) (2005) 367-421. MR 2178964, Zbl 1087.57011.

[28] G. C. Hruska. Geometric invariants of spaces with isolated flats. Topology 44(2) (2005) 441-458. MR 2114956, Zbl 1120.20046.

[29] G. C. Hruska \& B. Kleiner. Hadamard spaces with isolated flats. Geom. Topol. 9 (2005) 1501-1538. With an appendix by the authors and Mohamad Hindawi. MR 2175151, Zbl 1087.20034.

[30] C. Hummel \& V. Schroeder. Cusp closing in rank one symmetric spaces. Invent. Math. 123(2) (1996) 283-307. MR 1374201, Zbl 0860.53025.

[31] T. Januszkiewicz \& J. Świątkowski. Hyperbolic Coxeter groups of large dimension. Comment. Math. Helv. 78(3) (2003) 555-583. MR 1998394, Zbl 1068.20043.

[32] T. Januszkiewicz \& J. Świątkowski. Simplicial nonpositive curvature. Publ. Math. Inst. Hautes Études Sci. 104 (2006) 1-85. MR 2264834, Zbl 1143.53039.

[33] B. Kleiner \& B. Leeb. Rigidity of quasi-isometries for symmetric spaces and Euclidean buildings. Inst. Hautes Études Sci. Publ. Math. 86 (1998) 115-197. MR 1608566, Zbl 0910.53035.

[34] R. C. Lacher. Cell-like mappings. I. Pacific J. Math. 30 (1969) 717-731. MR 0251714, Zbl 0182.57601.

[35] J. Morgan \& G. Tian. Ricci flow and the Poincaré conjecture, volume 3 of: Clay Mathematics Monographs. American Mathematical Society, Providence, RI, 2007. MR 2334563, Zbl 1179.57045.

[36] J. Morgan \& G. Tian. Completion of the proof of the geometrization conjecture, 2008. preprint, arXiv:0809.4040.

[37] L. Mosher \& M. Sageev. Nonmanifold hyperbolic groups of high cohomological dimension, 1997. Preprint, available at http://andromeda.rutgers.edu/ mosher/.

[38] D. Osajda. Connectedness at infinity of systolic complexes and groups. Groups Geom. Dyn. 1(2) (2007)183-203. MR 2319456, Zbl 1128.20029.

[39] D. V. Osin. Peripheral fillings of relatively hyperbolic groups. Invent. Math. 167(2) (2007) 295-326. MR 2270456, Zbl 1116.20031.

[40] V. Schroeder. A cusp closing theorem. Proc. Amer. Math. Soc. 106(3) (1989) 797-802. MR 957267, Zbl 0678.53034.

[41] W. P. Thurston. Geometry and topology of three-manifolds. Princeton lecture notes available at http://www.msri.org/publications/books/gt3m/, 1980.

$$
\begin{array}{r}
\text { Graduate School of Information Sciences } \\
\text { Tohoku University } \\
\text { Sendai, 980-8579 } \\
\text { JaPAN } \\
\text { E-mail address: fujiwara@math.is.tohoku.ac.jp }
\end{array}
$$


Department of Mathematics UNIVERSITY AT BUFFALO

244 Mathematics Building Buffalo, NY 14260-2900

E-mail address: j399m@buffalo.edu 